\title{
Convergence of a structured metapopulation model to Levins's model
}

Received: 2 May 2003 / Revised version: 3 December 2003 /

Published online: $\mathbf{\square} 2004$ - (C) Springer-Verlag 2004

Abstract. We consider a structured metapopulation model describing the dynamics of a single species, whose members are located in separate patches that are linked through migration according to a mean field rule. Our main aim is to find conditions under which its equilibrium distribution is reasonably approximated by that of the unstructured model of Levins (1969). We do this by showing that the (positive) equilibrium distribution converges, as the carrying capacity of each population goes to infinity together with appropriate scalings on the other parameters, to a bimodal distribution, consisting of a point mass at 0 , together with a positive part which is closely approximated by a shifted Poisson centred near the carrying capacity. Under this limiting régime, we also give simpler approximate formulae for the equilibrium distribution. We conclude by showing how to compute persistence regions in parameter space for the exact model, and then illustrate all our results with numerical examples. Our proofs are based on Stein's method.

\section{Introduction}

A simple model describing the evolution of a metapopulation of animals, which are distributed over a number of patches that are linked by migration, was formulated by Levins [13] in 1969. He assumed all occupied patches to be equivalent, irrespective of the number of individuals present, and obtained the single logistic differential equation

$$
\frac{d p}{d t}=c_{L} p(1-p)-v_{L} p
$$

describing the behaviour of the system: here, $p=p(t)$ represents the proportion of occupied patches, $v_{L}$ is the extinction rate, and $c_{L}$ is the colonization rate per occupied patch. If $c_{L}>v_{L}$, the equations have a positive equilibrium, with proportion $\nu_{L} / c_{L}$ of patches empty; otherwise, the metapopulation becomes extinct. Generalization to structured metapopulation models, in which the numbers of individuals

A.D. Barbour: Angewandte Mathematik, Winterthurerstrasse 190, 8057 Zürich, Switzerland. e-mail: adb@amath.unizh.ch

A. Pugliese: Dipartimento di Matematica, Universitá di Trento, Via Sommarive 14, 38050 Povo (TN), Italy, e-mail: pugliese@science.unitn.it

Supported in part by Schweizer Nationalfonds Projekt Nrs 20-61753.00 and 20-67909.02

Supported in part by CNR of Italy under Grant n. 00.0142.ST74

Key words or phrases: Metapopulations - Levins's model - Convergence of equilibrium distribution - Poisson approximation

\begin{tabular}{|l|l|l|l|l|l}
$\mathbf{2}|\mathbf{8}| \mathbf{5}|\mathbf{0}| \mathbf{2}|\mathbf{7}| \mathbf{2} \mid \mathbf{B}$ & $\begin{array}{l}\text { Dispatch: 17/3/2004 } \\
\text { Total pages: } 33 \\
\text { Disk Received } \square \\
\text { Disk Used } \square\end{array}$ & $\begin{array}{l}\text { Journal: J. Math. Biol. } \\
\text { Not Used } \\
\text { Corrupted } \\
\text { Mismatch }\end{array}$ \\
\hline Jour. No $\mid$ Ms. No.
\end{tabular}


in the occupied patches also play a part, has been considered in Gyllenberg and Hanski [9], Casagrandi and Gatto [4], Metz and Gyllenberg [14] and Gyllenberg and Metz [10], among others.

Our starting point is the generalization studied by Arrigoni [1], who investigates a homogeneous stochastic mean field metapopulation model at the level of the individual. She proves that its behaviour approximates that of the infinite system of differential equations

$$
\left\{\begin{aligned}
p_{i}^{\prime}(t)= & -\left[\left(b_{i}+d_{i}+\gamma\right) i+v+\rho \gamma S(t)\right] p_{i}(t) \\
& +\left[b_{i-1}(i-1)+\rho \gamma S(t)\right] p_{i-1}(t) \\
& +\left[d_{i+1}+\gamma\right](i+1) p_{i+1}(t) ; \quad i \geq 1 \\
p_{0}^{\prime}(t)= & v\left(1-p_{0}(t)\right)+\left(d_{1}+\gamma\right) p_{1}(t)-\rho \gamma S(t) p_{0}(t) ; \\
\underline{p}(0)= & \underline{p}^{0},
\end{aligned}\right.
$$

with $S(t)=\sum_{j \geq 0} j p_{j}(t)$, when the number of patches becomes very large. Here, $p_{i}(t)$ denotes the proportion of patches that are occupied by $i$ individuals, $i \geq 0$, the parameters $b_{i}$ and $d_{i}$ represent the per capita birth and death rates in a patch occupied by $i$ individuals, $v$ denotes the catastrophe rate in each patch, and the migration rate is $\gamma$ per individual, with a proportion $\rho$ of migrants surviving to reach another patch. These equations are quite natural, and Arrigoni's results give a formal justification of their use; Metz and Gyllenberg [14] studied invasibility conditions for a very similar system, but with an extra compartment for dispersing individuals, and Casagrandi and Gatto [4] considered approximations to the system studied here, but allowing for migration rate $\gamma$ to depend on local abundance.

Unfortunately, the practical implications of the system (1.2) are not immediately visible. For instance, from the point of view of applications, it is interesting to ask how habitat loss (expressed in terms of the destruction of a proportion of the patches, or of the reduction of the carrying capacity in each) affects the persistence of the (meta)population. System (1.2), as it stands, provides no explicit formula relating persistence to the values of the parameters. In order to circumvent this problem, the relation between habitat loss and persistence has previously been studied by inserting habitat loss into Levins's original model (Nee and May [15]), by finding semi-empirical relations in stochastic versions of Levins' model (Hanski and Ovaskainen [11]), or by introducing low-dimensional approximations to (1.2) (Casagrandi and Gatto [6]) of 'moment-closure' type, without mathematical justification. However, even with these simplifications, the approximations still need to be calculated numerically.

In this paper, we are interested in improving our understanding of the long term behaviour of system (1.2), so that it can be used directly in addressing practical questions. We begin with the results of Barbour and Pugliese [3]; under the additional assumptions

(H1) $i b_{i}$ is concave and non-decreasing; $i d_{i}$ is convex and non-decreasing;

(H2) $\lim _{i \rightarrow \infty} b_{i}<\lim _{i \rightarrow \infty} d_{i}+v+\gamma(1-\rho)$, 
it is shown that system (1.2), which always has the extinction equilibrium $\pi=\pi_{e x}$ with $\pi\{0\}=1$ and $\pi\{i\}=0, i \geq 1$, also has exactly one other 'endemic' equilibrium probability distribution $\pi=\pi_{e n}$ satisfying the condition $\sum_{j \geq 0} j \pi\{j\}<\infty$, if a certain threshold condition given in (1.5) below is satisfied; and that then, if also $\lim _{i \rightarrow \infty} d_{i}<\infty$, there is global convergence to this equilibrium from any initial probability distribution $\lambda$ satisfying $0<\sum_{j \geq 0} j \lambda_{j}<\infty$. Moreover, the threshold condition (1.5) is represented by means of an explicit probabilistic formula (1.7). We exploit these features to obtain more detailed insight.

For instance, returning to the question of relating persistence to habitat loss, we are able to use the probabilistic form (1.7) of the threshold condition to compute persistence regions for the system in parameter space. We describe the procedure in Section 4, and illustrate the results in a specific, numerical example; the calculations can be made rather quickly. Hence, if such regions are to be numerically determined, there is actually no need for any of the previous approximations to (1.2). Note, however, that low-dimensional approximations can be used in conjunction with existing software for studying general bifurcation structure (see [6]).

Our primary concern is to find conditions under which Levins's [13] model can be recovered as a reasonable approximation to the model defined by (1.2), so that we can use the former as a simple but faithful description of the latter; an approach to this question based on simulation is also to be found in Keeling [12]. Intuitively, Levins' model should be adequate when all occupied patches are roughly equivalent. The qualitative conditions for this are easily understood. First, the birth and death process in a single patch should have a stable, long term quasi-equilibrium, or 'carrying capacity'. Secondly, the time taken to reach the carrying capacity from an initial state consisting of just one individual should typically be short compared to the time spent there before a catastrophe eliminates the population. With these two assumptions, suitably formalized, we use the generator approach to Stein's method to show that the number of individuals in a randomly chosen patch at equilibrium is either zero, or approximately at the carrying capacity, so that there are essentially just two distinct sorts of patches (Corollary 1 in Section 2); the analogues of Levins's extinction rate $v_{L}$ and colonization rate $c_{L}$ are also determined.

We are actually able to be more precise in the following results, proving an approximation to the part of the equilibrium distribution near the carrying capacity in a stronger sense than in the usual approximation with a normal distribution. We show that the conditional distribution over the occupied patches is closely approximated in total variation by a shifted Poisson distribution having (almost) the same mean and variance, and that the approximation error is of the same asymptotic order as the best that could possibly be obtained for any usual approximation with the normal distribution: see Remark 1. We accomplish this by using the Stein-Chen method, together with a local linearization of the generator of an approximating birth and death process.

In order to determine the parameters $v_{L}$ and $c_{L}$ of the Levins model approximating (1.2), it is necessary to find the positive solution $s^{*}$ of a rather complicated fixed point equation, which is given in (1.6) below. In Section 3, under the same 
conditions as are used in Section 2 to justify the Levins approximation, we derive relatively simple approximations to $s^{*}$, and show that it then does not introduce large errors into the approximation of the equilibrium distribution if these approximations are used in place of $s^{*}$. We illustrate the efficacy of our approximations in Section 4, in a particular instance of the model and for a range of parameter values. These results also indicate that the qualitative picture derived from the earlier, approximate treatments is reasonable, even if the numerical values differ in detail.

We conclude the introduction by stating the threshold theorem proved in (1.2). A key observation is that, if the quantity $S(t)$ in (1.2) is replaced by any fixed value $s \geq 0$, then the equations become the Kolmogorov forward differential equations for an immigration, birth, death and catastrophe process $Z^{(s)}$ with transition rates

$$
\begin{array}{rrc}
j \rightarrow j+1 & \text { at rate } & j b_{j}+\rho \gamma s \\
j \rightarrow j-1 & \text { at rate } & j\left(d_{j}+\gamma\right) \\
j \rightarrow 0 & \text { at rate } & \nu .
\end{array}
$$

If $\lim _{i \rightarrow \infty} b_{i}-\lim _{i \rightarrow \infty} d_{i}-\gamma<v$, true a fortiori if (H2) holds, then the process $Z^{(s)}$ has an equilibrium distribution $\pi^{(s)}$ which satisfies

$$
G(s):=\sum_{j \geq 0} j \pi^{(s)}\{j\}<\infty ;
$$

furthermore, under Assumptions (H1) and (H2), the function $G$ is increasing and concave, and, if the threshold condition

$$
G^{\prime}(0)>1
$$

is satisfied, then the differential equations (1.2) have $\pi^{\left(s^{*}\right)}$ as endemic equilibrium solution, where $s^{*}$ is the unique positive solution to the fixed point equation

$$
s=G(s) .
$$

Furthermore, the quantity $G^{\prime}(0)$ has a probabilistic representation:

$$
G^{\prime}(0)=\rho \gamma \int_{0}^{\infty} \mathbb{E}^{(1)} Z_{t}^{(0)} d t .
$$

Thus $G^{\prime}(0)$ is the average of the total number of migrants surviving to reach another patch, emanating from a patch initially colonized by a single immigrant, up to the time of population extinction in that patch, and when no other migrations into that patch are allowed. This number may be interpreted as a reproduction number for colonizers of an empty habitat, analogous to the reproduction numbers used in epidemic models (Diekmann et al. [8]). A similar condition has been given by Chesson [7] and by Casagrandi and Gatto [5]; see also Metz and Gyllenberg [14]. 


\section{Approximations to the invariant distribution}

For Levins's [13] model to be a useful approximation to the solutions to (1.2), we should require the single patch dynamics to have a stable, long term quasi-equilibrium, and the time to reach quasi-equilibrium from a single founder individual to be short compared to the average time between catastrophes. More formally, we consider a sequence of models as in (1.2), indexed by a parameter $K$, which we think of as 'large'. We first suppose that $b_{i}=b(i / K), d_{i}=d(i / K)$ for fixed functions $b, d: \mathbb{R}_{+} \rightarrow \mathbb{R}_{+}$which satisfy

1. $x b(x)$ is nondecreasing and concave.

2. $x d(x)$ is nondecreasing and convex.

3. $b(1)=d(1)$.

It is also convenient to assume that both $b$ and $d$ are twice continuously differentiable, that $b(0)>d(0)$, and that $b^{\prime}(0)$ and $d^{\prime}(0)$ are both finite. These conditions ensure that the population size, if positive, remains close to the value $K$, if $K$ is large and $\gamma$ is small, fulfilling the first of the natural conditions, that of a stable quasi-equilibrium.

The choice of 1 for the value of $x$ at which $b(x)=d(x)$ merely represents a convenient standardization for $K$. This is because, if $b(c)=d(c)$ for some $c \neq 1$, one could replace the functions $b$ and $d$ by the functions $b_{c}$ and $d_{c}$ defined by $b_{c}(x):=b(c x), d_{c}(x):=d(c x)$ for all $x$, and then set $K_{c}:=c K$; this would yield $b_{i}=b_{c}\left(i / K_{c}\right)$ and $d_{i}=d_{c}\left(i / K_{c}\right)$ as an equivalent representation of the birth and death rates $b_{i}$ and $d_{i}$, but now with $b_{c}(1)=d_{c}(1)$. Thus Condition 3 is not essential in what follows, and can be dispensed with, provided that, in the error bounds that we establish, $K$ is replaced by $c K$.

The parameters $\rho=\rho(K), \gamma=\gamma(K)$ and $v=v(K)$ we allow to depend on $K$. We prove qualitative bounds for our approximations which are uniform for all $\rho \leq 1$, and also for all $\gamma \leq \gamma_{0}$, where $\gamma_{0}$ is fixed but chosen to be small enough; the bounds are expressed in terms of the parameters $v$ and $K$, and become small as $K \rightarrow \infty$ so long as $v \log K \rightarrow 0$ as $K \rightarrow \infty$. With this in mind, we actually consider our bounds only under the condition that

\section{4. $v \log K \leq \eta_{0}$,}

where $\eta_{0}$ depends only on $b, d$ and $\gamma_{0}$. Condition 4 requires that the average time $v^{-1}$ between catastrophes be sufficiently much larger than the time spent between an initial immigration and first reaching quasi-equilibrium, which is of order $O(\log K)$.

We do not give an explicit expression for $\eta_{0}$, though one could be derived from inspection of the proof of Lemma 3. For $\gamma_{0}$, we assume at least that it is small enough to ensure that the following conditions are satisfied:

5a. The solution $x_{-}<1$ to $b(x)=d(x)+\gamma_{0}$ satisfies $x_{-}>3 / 4$.

5b. The solution $x_{+}>1$ to $b(x)+2 x^{-1} \gamma_{0}=d(x)$ satisfies $x_{+}<5 / 4$.

5c. $\gamma_{0}<\frac{1}{2}\{b(5 / 8)-d(5 / 8)\}$.

5d. $c_{0}:=\left(d(1 / 2)+\gamma_{0}\right) / b(1 / 2)<1 ; \quad c_{1}:=\left(b(3 / 2)+2 \gamma_{0}\right) / d(3 / 2)<1$.

5e. $\gamma_{0}<\frac{1}{2} x_{-}\left(d^{\prime}\left(x_{-}\right)-b^{\prime}\left(x_{-}\right)\right)$. 
Note that

$$
x_{-}\left\{d^{\prime}\left(x_{-}\right)-b^{\prime}\left(x_{-}\right)\right\}=\frac{d}{d x}\left[x\left\{d(x)+\gamma_{0}-b(x)\right\}\right]_{x=x_{-}}>0
$$

is implied by Conditions 1 and 2 and because $b(0)>d(0)$. It will emerge that the probability of a patch being empty is roughly of order $v /(\rho \gamma K)$, so that when $\rho=1$, for instance, Conditions $5 \mathrm{a}-5 \mathrm{e}$ are automatic if this probability is not to be extremely small.

Under these circumstances, we wish to show that the endemic equilibrium distribution $\pi_{e n}$ of (1.2) is close to that suggested by Levins's model; that is, we wish to show that $\pi$ concentrates almost all of its mass either at 0 or near some fixed positive value, and that, setting $\pi\{0\}=: v_{L} / c_{L}$, as in Levins's model, we obtain a plausible interpretation for his colonization rate $c_{L}$.

Now if $G^{\prime}(0)>1$, the equilibrium distribution $\pi_{e n}$ is just the distribution $\pi^{\left(s^{*}\right)}$ for $s^{*}$ the solution to (1.6). Our main task is therefore to show that, under Conditions $1-5$ above, for large $K$ and for any $s$, the distribution $\pi^{(s)}$ is concentrated near two points, one of which is 0 . To do this, we start by investigating $\pi^{0(s)}$, the equilibrium distribution of the immigration, birth and death process with the same birth, death and immigration rates as $Z^{(s)}$, except for having the death and migration rates from the state 1 set to zero, and with $v=0$. This distribution is our candidate for approximating the part of $\pi^{(s)}$ off 0 . We show that $\pi^{0(s)}$ is concentrated near $\lfloor K \kappa\rfloor$, where $\kappa$ is the positive solution of

$$
b(\kappa)+\rho \gamma s /(K \kappa)=d(\kappa)+\gamma,
$$

close to 1 if $\gamma$ is small. In more detail, we show that $\pi^{0(s)}$ is very close to a shifted Poisson distribution, whose spread, of order $O\left(K^{1 / 2}\right)$, is small in comparison to $\lfloor K \kappa\rfloor$. We then prove that $\pi^{(s)}$ is close to

$$
\pi^{\prime(s)}:=\pi^{(s)}\{0\} \Delta_{\{0\}}+\left(1-\pi^{(s)}\{0\}\right) \pi^{0(s)} .
$$

While examining the properties of the distribution $\pi^{0(s)}$, we temporarily suppress the dependence on both $s$ and $K$, and define, for all $i \geq 1$,

$$
\begin{aligned}
& \lambda_{i}:=i b_{i}+\rho \gamma s=i b(i / K)+\rho \gamma s \quad \text { and } \\
& \mu_{i}:=i\left(d_{i}+\gamma\right)=i(d(i / K)+\gamma) ;
\end{aligned}
$$

then $\pi^{0}\{i\}$ can be expressed in terms of $\lambda_{i}$ and $\mu_{i}, i \geq 1$, as

$$
\pi^{0}\{i\}:=\frac{w_{i}}{\sum_{j \geq 1} w_{j}}, \quad \text { where } \quad w_{i}:=\frac{1}{\mu_{i}} \prod_{j=1}^{i-1}\left(\frac{\lambda_{j}}{\mu_{j}}\right) .
$$

Note that, from (2.1), $\lambda_{j} \geq \mu_{j}$ when $j \leq\lfloor K \kappa\rfloor$ and $\lambda_{j}<\mu_{j}$ when $j \geq\lfloor K \kappa\rfloor+1$, and that, for $0 \leq s \leq 2 K$,

$$
|1-\kappa| \leq 1 / 4
$$


in view of Conditions $5 \mathrm{a}$ and $5 \mathrm{~b}$. Thus we have $\mu_{i} w_{i} \leq \mu_{i+1} w_{i+1}$ in $i \leq\lfloor K \kappa\rfloor$ and $\mu_{i} w_{i}>\mu_{i+1} w_{i+1}$ in $i>\lfloor K \kappa\rfloor$, with geometrically fast decay far enough away from $i=\lfloor K \kappa\rfloor$ in either case, so that the probability distribution $\pi^{0}$ is strongly concentrated around $\lfloor K \kappa\rfloor$. This is the substance of the following lemma; it makes precise the assertion that the spread of $\pi^{0}$ around $\lfloor K \kappa\rfloor$ is of order $O\left(K^{1 / 2}\right)$.

Lemma 1. With the above definitions, given any $0<\eta \leq 1 / 2$, there exists an $\alpha=\alpha(\eta)>0$ such that

1. $\pi^{0}[1,\lfloor(1-\eta) K \kappa\rfloor]=O\left(K^{-1 / 2} e^{-\alpha K}\right)$;

2. $\pi^{0}[\lfloor(1+\eta) K \kappa\rfloor, \infty)=O\left(K^{-1 / 2} e^{-\alpha K}\right)$.

3. $\sum_{j \geq\lfloor 2 K \kappa\rfloor+1} j \pi^{0}\{j\}=O\left(\sqrt{K} e^{-\alpha K}\right)$

4. $\sum_{j=\lfloor K \kappa / 2\rfloor+1}^{\lfloor 2 K \kappa\rfloor}|j-\lfloor K \kappa\rfloor|^{r} \pi^{0}\{j\}=O\left(K^{r / 2}\right)$ for all $r \geq 1$.

All these bounds hold uniformly in $\rho \leq 1, \gamma \leq \gamma_{0}$ and in $s \leq 2 K$.

Proof. Because $b$ is non-increasing and $d$ is non-decreasing, we have

$$
\begin{aligned}
\left(\lambda_{\lfloor K \kappa / 2\rfloor}-\mu_{\lfloor K \kappa / 2\rfloor}\right) /\lfloor K \kappa / 2\rfloor & \geq\left(b(5 / 8)-d(5 / 8)-\gamma_{0}\right) \\
& >\frac{1}{2}(b(5 / 8)-d(5 / 8))>0,
\end{aligned}
$$

by (2.4) and Condition 5c. Hence, by the concavity of $x b(x)$ and the convexity of $x d(x)$, it follows that

$$
\begin{array}{ll}
\lambda_{j}-\mu_{j} \geq(K \kappa-j) D^{\prime} & \text { in } \quad\lfloor K \kappa / 2\rfloor \leq j \leq\lfloor K \kappa\rfloor ; \\
\mu_{j}-\lambda_{j} \geq(j-K \kappa) D^{\prime} \quad \text { in } \quad j>\lfloor K \kappa\rfloor,
\end{array}
$$

where $D^{\prime}=\frac{1}{2}(b(5 / 8)-d(5 / 8))\lfloor K \kappa / 2\rfloor /(K \kappa-\lfloor K \kappa / 2\rfloor)$; since, from (2.4),

$$
\frac{\lfloor K \kappa / 2\rfloor}{K \kappa-\lfloor K \kappa / 2\rfloor} \geq 1-\frac{4}{2 K \kappa+1} \geq 1-\frac{8}{3 K+2} \geq \frac{1}{2}
$$

in $K \geq 5$, we then have

$$
\begin{array}{ll}
\lambda_{j}-\mu_{j} \geq(K \kappa-j) D & \text { in } \quad\lfloor K \kappa / 2\rfloor \leq j \leq\lfloor K \kappa\rfloor ; \\
\mu_{j}-\lambda_{j} \geq(j-K \kappa) D & \text { in } \quad j>\lfloor K \kappa\rfloor,
\end{array}
$$

with $D=\frac{1}{4}(b(5 / 8)-d(5 / 8))$. Similarly, noting that

$$
(\mu\lfloor 2 K \kappa\rfloor-\lambda\lfloor 2 K \kappa\rfloor) /\lfloor 2 K \kappa\rfloor \leq\left\{d(5 / 2)-b(5 / 2)+\gamma_{0}\right\},
$$

and that, from (2.4),

$$
\frac{\lfloor 2 K \kappa\rfloor}{\lfloor 2 K \kappa\rfloor-K \kappa} \leq 2+\frac{1}{K \kappa-1} \leq 2+\frac{4}{3 K-4} \leq 3
$$

in $K \geq 3$, it follows that

$$
\begin{array}{ll}
\lambda_{j}-\mu_{j} \leq(K \kappa-j) D_{1} \quad \text { in } \quad 1 \leq j \leq\lfloor K \kappa\rfloor ; \\
\mu_{j}-\lambda_{j} \leq(j-K \kappa) D_{1} \quad \text { in } \quad\lfloor K \kappa\rfloor<j \leq\lfloor 2 K \kappa\rfloor,
\end{array}
$$

with $D_{1}=3\left\{d(5 / 2)-b(5 / 2)+\gamma_{0}\right\}$. 
First, consider the range $\lfloor K \kappa / 2\rfloor \leq j \leq\lfloor K \kappa\rfloor$. Then it follows from (2.5) that

$$
\frac{\mu_{j}}{\lambda_{j}}=1-\frac{\lambda_{j}-\mu_{j}}{\lambda_{j}} \leq \exp \left\{-D\left(\frac{K \kappa-j}{\lambda_{j}}\right)\right\},
$$

and hence, since $\lambda_{l}$ is increasing in $l$, we have

$$
\prod_{l=j}^{\lfloor K \kappa\rfloor} \frac{\mu_{l}}{\lambda_{l}} \leq \exp \left\{-\frac{1}{2} D \lambda_{\lfloor K \kappa\rfloor}^{-1}(\lfloor K \kappa\rfloor-j)(\lfloor K \kappa\rfloor-j+1)\right\} .
$$

Thus

$$
\hat{w}_{j}:=\frac{1}{\mu_{j}} \prod_{l=j}^{\lfloor K \kappa\rfloor} \frac{\mu_{l}}{\lambda_{l}} \leq \frac{1}{\mu_{j}} \exp \left\{-\frac{1}{2} D \lambda_{\lfloor K \kappa\rfloor}^{-1}(\lfloor K \kappa\rfloor-j)(\lfloor K \kappa\rfloor-j+1)\right\}
$$

in $\lfloor K \kappa / 2\rfloor \leq j \leq\lfloor K \kappa\rfloor$. Then, in similar fashion, starting from (2.7), we have

$$
\frac{\mu_{j}}{\lambda_{j}} \geq \max \left\{0,1-D_{1}\left(\frac{K \kappa-j}{\lambda_{j}}\right)\right\},
$$

so that

$$
\begin{aligned}
\hat{w}_{j} & \geq \frac{1}{\mu_{j}}\left\{1-\sum_{l=j}^{\lfloor K \kappa\rfloor} D_{1}\left(\frac{K \kappa-l}{\lambda_{l}}\right)\right\} \\
& \geq \frac{1}{\mu_{j}}\left(1-\frac{1}{2} D_{1} \lambda_{j}^{-1}(\lfloor K \kappa\rfloor-j+1)(\lfloor K \kappa\rfloor-j+2)\right) \text { in }\lfloor K \kappa / 2\rfloor \leq j \leq\lfloor K \kappa\rfloor ;
\end{aligned}
$$

this implies that, for those $j$ in the interval

$$
\lfloor K \kappa\rfloor \geq j \geq\lfloor K \kappa\rfloor-\max \left\{0,\left(\left\lfloor\sqrt{D_{1}^{-1} \lambda_{\lfloor K \kappa / 2\rfloor}}\right\rfloor-2\right)\right\},
$$

we have $\hat{w}_{j} \geq 1 / 2 \mu_{j}$. Now

$$
\lambda_{\lfloor K \kappa / 2\rfloor} \geq\lfloor K \kappa / 2\rfloor b(\kappa / 2) \geq\lfloor K \kappa / 2\rfloor b(3 / 4) \geq\lfloor 3 K / 8\rfloor \gamma_{0},
$$

by (2.4) and Condition 5a, and so

$$
\left\lfloor\sqrt{D_{1}^{-1} \lambda_{\lfloor K \kappa / 2\rfloor}}\right\rfloor-2 \geq c_{*} \sqrt{K}
$$

for some $c_{*}>0$ and for all $K \geq K_{0}$ large enough. Then, for $j \leq\lfloor K \kappa\rfloor$, we have

$$
\mu_{\lfloor K \kappa\rfloor} \leq K \kappa(d(\kappa)+\gamma) \leq K \kappa\left(b(\kappa)+2 \gamma_{0} / \kappa\right) \leq K\left\{(5 / 4) b(5 / 4)+2 \gamma_{0}\right\},
$$

again by (2.4) and the properties of $b$ and $d$. Hence, in the sum $\sum_{j \geq 1} \hat{w}_{j}$, there are at least $c_{*} \sqrt{K}$ terms each of size at least $\left[2 K\left\{(5 / 4) b(5 / 4)+2 \gamma_{0}\right\}\right]^{-1}$, implying that

$$
\sum_{j \geq 1} \hat{w}_{j} \geq C_{1} K^{-1 / 2}
$$


for some $C_{1}>0$, uniformly for all $\gamma \leq \gamma_{0}$ and $0 \leq s \leq 2 K$. Also,

$$
\frac{\hat{w}_{j-1}}{\hat{w}_{j}}=\frac{\mu_{j}}{\lambda_{j-1}} \leq \frac{j \mu_{j}}{(j-1) \lambda_{j}}
$$

and $\mu_{j} / \lambda_{j}$ is increasing in $j \leq\lfloor K \kappa\rfloor$, so that, since also $j / l \leq j-l+1$ in $1 \leq l \leq j$, we have

$$
\sum_{l=1}^{j} \hat{w}_{l} \leq \frac{1}{\mu_{j}}\left(\frac{\lambda_{j}}{\lambda_{j}-\mu_{j}}\right)^{2} \exp \left\{-\frac{1}{2} D \lambda_{\lfloor K \kappa\rfloor}^{-1}(\lfloor K \kappa\rfloor-j)(\lfloor K \kappa\rfloor-j+1)\right\}
$$

for $\lfloor K \kappa / 2\rfloor \leq j \leq\lfloor K \kappa\rfloor$. In particular, taking $j=\lfloor(1-\eta) K \kappa\rfloor$ in (2.11), we have

$$
\sum_{l=1}^{\lfloor(1-\eta) K \kappa\rfloor} \hat{w}_{l}=O\left(K^{-1} e^{-\alpha_{1} K}\right)
$$

for some $\alpha_{1}=\alpha_{1}(\eta)>0$.

For the range $\lfloor K \kappa\rfloor+1 \leq j \leq\lfloor 2 K \kappa\rfloor$, a similar argument based on (2.6) shows that

$$
\hat{w}_{j}:=\frac{1}{\mu_{j}} \prod_{l=\lfloor K \kappa\rfloor+1}^{j-1} \frac{\lambda_{l}}{\mu_{l}} \leq \frac{1}{\mu_{j}} \exp \left\{-\frac{1}{2} D \mu_{\lfloor 2 K \kappa\rfloor}^{-1}(j-\lfloor K \kappa\rfloor)(j-\lfloor K \kappa\rfloor-1)\right\},
$$

and that therefore, since

$$
\frac{\hat{w}_{j+1}}{\hat{w}_{j}}=\frac{\lambda_{j}}{\mu_{j+1}} \leq \frac{\lambda_{j+1}}{\mu_{j+1}}
$$

and $\lambda_{j} / \mu_{j}$ is decreasing in $j>\lfloor K \kappa\rfloor$, it follows that

$$
\sum_{l=\lfloor(1+\eta) K \kappa\rfloor+1}^{\infty} \hat{w}_{l}=O\left(K^{-1} e^{-\alpha_{2} K}\right)
$$

again for some $\alpha_{2}=\alpha_{2}(\eta)>0$. But, for any set $A \subset \mathbb{N}$,

$$
\pi^{0}\{A\}=\left(\sum_{j \in A} \hat{w}_{j}\right) /\left(\sum_{j \geq 1} \hat{w}_{j}\right),
$$

so that parts 1 and 2 follow from (2.10), (2.12) and (2.15).

Next, from(2.14), for $j \geq\lfloor 2 K \kappa\rfloor$, we have

$$
\hat{w}_{j} \leq \hat{w}_{\lfloor 2 K \kappa\rfloor}\left(\lambda_{\lfloor 2 K \kappa\rfloor+1} / \mu_{\lfloor 2 K \kappa\rfloor+1}\right)^{j-\lfloor 2 K \kappa\rfloor},
$$


in which, from (2.6),

$$
\frac{\lambda_{\lfloor 2 K \kappa\rfloor+1}}{\mu_{\lfloor 2 K \kappa\rfloor+1}} \leq 1-\frac{1}{2} D\left\{d(2 \kappa)+\gamma_{0}\right\}^{-1} \leq 1-\frac{1}{2} D\left\{d(5 / 2)+\gamma_{0}\right\}^{-1}=: c_{1}<1 ;
$$

part 3 is now immediate. For part 4, we just use (2.16), (2.10), (2.9) and (2.13) to give an upper bound of the required order for the sum $\sum_{j=\lfloor K \kappa / 2\rfloor+1}^{\lfloor 2 K \kappa\rfloor}|j-\lfloor K \kappa\rfloor|^{r} \pi^{0}\{j\}$.

The previous lemma gives broad bounds on the concentration of $\pi^{0}$ around $\lfloor K \kappa\rfloor$. The next theorem shows that $\pi^{0}$ is actually rather close to a shifted Poisson distribution, in the sense of total variation. Once again, order statements are understood to be uniform in $\rho \leq 1, \gamma \leq \gamma_{0}$ and in $s \leq 2 K$.

Theorem 1. Let $B=B^{(s)}:=\left\lfloor K \beta_{1} / \beta_{2}\right\rfloor$, where

$$
\beta_{1}=\beta_{1}^{(s)}:=\kappa b(\kappa)+\rho \gamma s / K \text { and } \beta_{2}=\beta_{2}^{(s)}:=\kappa\left(d^{\prime}(\kappa)-b^{\prime}(\kappa)\right)+\rho \gamma s /(K \kappa) \text {. }
$$

Then $d_{T V}\left(\pi^{0}, \Delta_{\{\lfloor K \kappa\rfloor\}} * \widehat{\mathrm{Po}}(B)\right)=O\left(K^{-1 / 2}\right)$, where, for integer $\lambda$, $\widehat{\mathrm{Po}}(\lambda)$ denotes the centred Poisson distribution with variance $\lambda, \Delta_{\{j\}}$ denotes the point mass at $j$ and $*$ denotes convolution.

Proof. An essential feature of the Stein-Chen method is that, for each $C \subset \mathbf{Z}_{+}$, the function $g_{C}$ solving

$$
1_{C}(j)-\operatorname{Po}(\lambda)\{C\}=\lambda g_{C}(j+1)-j g_{C}(j), \quad j \geq 0,
$$

satisfies the bounds

$$
\|g\| \leq \min \left\{1, \lambda^{-1 / 2}\right\} ; \quad\|\Delta g\| \leq \min \left\{1, \lambda^{-1}\right\}:
$$

see Barbour [2], p.84. Hence, if $W$ is any (possibly negative) integer valued random variable and $C \in \mathbf{Z}_{+}$, and if $0<\eta \leq 1$, we can use (2.17) when $|W-\lambda| \leq \eta \lambda$ to obtain

$$
\begin{aligned}
& |\mathbb{P}[W \in C]-\operatorname{Po}(\lambda)\{C\}|=\left|\mathbb{E}\left\{1_{C}(W)-\operatorname{Po}(\lambda)\{C\}\right\}\right| \\
& \quad \leq\left|\mathbb{E}\left\{\left(\lambda g_{C}(W+1)-W g_{C}(W)\right) I[|W-\lambda| \leq \eta \lambda]\right\}\right|+\mathbb{P}[|W-\lambda|>\eta \lambda] .
\end{aligned}
$$

Thus, if it can be shown that

$$
|\mathbb{E}\{(\lambda g(W+1)-W g(W)) I[|W-\lambda| \leq \eta \lambda]\}| \leq \varepsilon_{1}
$$

for any function $g$ which is bounded as above, and that

$$
\mathbb{P}[|W-\lambda|>\eta \lambda] \leq \varepsilon_{2},
$$

then it follows that

$$
d_{T V}(\mathcal{L}(W), \operatorname{Po}(\lambda)):=\sup _{C \subset \mathbf{Z}}|P[W \in C]-\operatorname{Po}(\lambda)\{C\}| \leq \varepsilon_{1}+\varepsilon_{2} .
$$


We apply this estimate with $\lambda=B$ to a random variable $V-\lfloor K \kappa\rfloor+B$, where $V \sim \pi^{0}$

Since $\pi^{0}$ is just the equilibrium distribution for the birth and death process on $\mathbb{N}$ with birth rates $\lambda_{j}, j \geq 1$, and death rates $\mu_{j}, j \geq 2$, we have

$$
\mu_{J} g(J) \pi^{0}\{J\}+\sum_{j=J}^{N}\left\{\lambda_{j} g(j+1)-\mu_{j} g(j)\right\} \pi^{0}\{j\}-\lambda_{N} g(N+1) \pi^{0}\{N\}=0
$$

for all bounded $g$ and all $1 \leq J<N$, by detailed balance. Now, for $j=\lfloor K \kappa\rfloor+l$, we have the expansion

$$
\begin{aligned}
\lambda_{\lfloor K \kappa\rfloor+l}= & (\lfloor K \kappa\rfloor+l) b\left(\frac{\lfloor K \kappa\rfloor+l}{K}\right)+\rho \gamma s \\
= & \lfloor K \kappa\rfloor b\left(\frac{\lfloor K \kappa\rfloor}{K}\right)+\rho \gamma s+l\left\{b\left(\frac{\lfloor K \kappa\rfloor}{K}\right)+\frac{\lfloor K \kappa\rfloor}{K} b^{\prime}\left(\frac{\lfloor K \kappa\rfloor}{K}\right)\right\} \\
& +O\left(K^{-1} l^{2}\right),
\end{aligned}
$$

uniformly in $|l| \leq\lfloor K \kappa\rfloor-1$; similarly,

$$
\begin{aligned}
\mu_{\lfloor K \kappa\rfloor+l}=\lfloor K \kappa\rfloor & \left\{d\left(\frac{\lfloor K \kappa\rfloor}{K}\right)+\gamma\right\}+l\left\{d\left(\frac{\lfloor K \kappa\rfloor}{K}\right)+\gamma+\frac{\lfloor K \kappa\rfloor}{K} d^{\prime}\left(\frac{\lfloor K \kappa\rfloor}{K}\right)\right\} \\
+ & O\left(K^{-1} l^{2}\right),
\end{aligned}
$$

in the same range of $l$. Thus it follows that

$$
\begin{aligned}
& \lambda_{\lfloor K \kappa\rfloor+l} g(\lfloor K \kappa\rfloor+l+1)-\mu_{\lfloor K \kappa\rfloor+l} g(\lfloor K \kappa\rfloor+l) \\
&=K \beta_{1}\{g(\lfloor K \kappa\rfloor+l+1)-g(\lfloor K \kappa\rfloor+l)\}-l \beta_{2} g(\lfloor K \kappa\rfloor+l) \\
&+O((1+|l|)\|\Delta g\|)+O\left(K^{-1}|l|(1+|l|)\|g\|\right),
\end{aligned}
$$

uniformly in $|l| \leq\lfloor K \kappa\rfloor-1$. Hence we have

$$
\begin{array}{rl}
\lambda\lfloor K \kappa\rfloor+l & g(\lfloor K \kappa\rfloor+l+1)-\mu_{\lfloor K \kappa\rfloor+l} g(\lfloor K \kappa\rfloor+l) \\
=\beta_{2} & \left\{\left(K \beta_{1} / \beta_{2}\right) g(\lfloor K \kappa\rfloor+l+1)-\left(l+K \beta_{1} / \beta_{2}\right) g(\lfloor K \kappa\rfloor+l)\right\} \\
& +O((1+|l|)\|\Delta g\|)+O\left(K^{-1}|l|(1+|l|)\|g\|\right) \\
=\beta_{2} & \{B g(j+1)-(j-A) g(j)\} \\
+ & O((1+|l|)\|\Delta g\|)+O\left(K^{-1}|l|(1+|l|)\|g\|\right),
\end{array}
$$

uniformly in $1 \leq j \leq 2\lfloor K \kappa\rfloor-1$, with $B=\left\lfloor K \beta_{1} / \beta_{2}\right\rfloor$ as before, $A=\lfloor K \kappa\rfloor-B$ and with $j=\lfloor K \kappa\rfloor+l$. Note that, under $\pi^{0}$, the 'typical' values of $l$ satisfy $|l|=O\left(K^{1 / 2}\right)$.

Suppose that $0<\eta \leq 1$ satisfies $B \eta \leq\lfloor K \kappa\rfloor-1$. Then it follows from (2.21) by taking $\tilde{g}(j)=g(j+\bar{A})$ that, for all $\tilde{g}$ satisfying $\|\tilde{g}\| \leq B^{-1 / 2}$ and $\|\Delta \tilde{g}\| \leq B^{-1}$, 
we have

$$
\begin{aligned}
& \sum_{m=B-\lfloor\eta B\rfloor}^{B+\lfloor\eta B\rfloor} \pi^{0}\{m+A\}(B \tilde{g}(m+1)-m \tilde{g}(m)) \\
& \quad=\sum_{j=K_{B}^{-}}^{K_{B}^{+}} \pi^{0}\{j\}\{B g(j+1)-(j-A) g(j)\} \\
& \quad=\frac{1}{\beta_{2}} \sum_{j=K_{B}^{-}}^{K_{B}^{+}} \pi^{0}\{j\}\left\{\lambda_{j} g(j+1)-\mu_{j} g(j)\right\} \\
& +O\left(\sum_{j=K_{B}^{-}}^{K_{B}^{+}} \pi^{0}\{j\}\left\{K^{-1}|j-\lfloor K \kappa\rfloor|+K^{-3 / 2}(j-\lfloor K \kappa\rfloor)^{2}\right\}\right),
\end{aligned}
$$

where $K_{B}^{+}:=\lfloor K \kappa\rfloor+\lfloor\eta B\rfloor$ and $K_{B}^{-}:=\lfloor K \kappa\rfloor-\lfloor\eta B\rfloor$. To estimate (2.22), first use (2.20) to get

$$
\begin{aligned}
& \left|\sum_{j=K_{B}^{-}}^{K_{B}^{+}} \pi^{0}\{j\}\left\{\lambda_{j} g(j+1)-\mu_{j} g(j)\right\}\right| \\
& \quad=\left|\lambda_{K_{B}^{+}} g\left(K_{B}^{+}+1\right) \pi^{0}\left\{K_{B}^{+}\right\}-\mu_{K_{B}^{-}} g\left(K_{B}^{-}\right) \pi^{0}\left\{K_{B}^{-}\right\}\right| \\
& \leq 2 \beta_{1} K\|g\| \pi^{0}\left\{K_{B}^{+}\right\}+\beta_{1} K\|g\| \pi^{0}\left\{K_{B}^{-}\right\},
\end{aligned}
$$

where the last line follows because $x b(x)$ is concave and $x d(x)$ is increasing, and from (2.1). Hence, in view of Lemma 1.1 and the bound $\|g\| \leq B^{-1 / 2}$, this quantity is exponentially small with $K$, and hence of order $O\left(K^{-1 / 2}\right)$, uniformly in $\rho \leq 1$, $\gamma \leq \gamma_{0}$ and $0 \leq s \leq 2 K$, for any fixed $\eta>0$. Also, from Lemma 1.4, the order term in (2.22) is of order $O\left(K^{-1 / 2}\right)$ if

$$
\lfloor K \kappa / 2\rfloor \leq\lfloor K \kappa\rfloor-\lfloor\eta B\rfloor<\lfloor K \kappa\rfloor+\lfloor\eta B\rfloor \leq\lfloor 2 K \kappa\rfloor .
$$

Now $\beta_{1} /\left(\kappa \beta_{2}\right)$ is bounded below by

$$
C_{-}:=b(5 / 4) /\left\{x_{+}\left(d^{\prime}\left(x_{+}\right)-b^{\prime}\left(x_{+}\right)\right)+2 \gamma_{0} / x_{+}\right\},
$$

and above by

$$
C_{+}:=\left\{b(3 / 4)+8 \gamma_{0} / 3\right\} /\left\{x_{-}\left(d^{\prime}\left(x_{-}\right)-b^{\prime}\left(x_{-}\right)\right)\right\},
$$

uniformly in $\rho \leq 1, \gamma \leq \gamma_{0}$ and $0 \leq s \leq 2 K$, since $b(x)+\rho \gamma s /(K x)$ is non-increasing in $x$ and

$$
\kappa\left\{d^{\prime}(\kappa)-b^{\prime}(\kappa)\right\}+\rho \gamma s /(K \kappa)=\left.\frac{d}{d x}\{x(d(x)+\gamma-b(x))\}\right|_{x=\kappa}
$$


is non-decreasing in $\kappa$, so that

$$
\begin{aligned}
x_{-}\left(d^{\prime}\left(x_{-}\right)-b^{\prime}\left(x_{-}\right)\right) & \leq \kappa\left\{d^{\prime}(\kappa)-b^{\prime}(\kappa)\right\}+\rho \gamma s /(K \kappa) \\
& \leq x_{+}\left(d^{\prime}\left(x_{+}\right)-b^{\prime}\left(x_{+}\right)\right)+2 \gamma_{0} / x_{+} .
\end{aligned}
$$

Thus we can ensure that (2.24) is satisfied for all $\rho \leq 1, \gamma \leq \gamma_{0}$ and $0 \leq s \leq 2 K$ if we choose $\eta=1 /\left(4 C_{+}\right)$, provided that $\lfloor K \kappa\rfloor \geq 1$; hence it follows from the previous paragraph that

$$
\sum_{m=B-\lfloor\eta B\rfloor}^{B+\lfloor\eta B\rfloor} \pi^{0}\{m+A\}(B g(m+1)-m g(m))=O\left(K^{-1 / 2}\right),
$$

uniformly in $0 \leq s \leq 2 K, \rho \leq 1$ and $\gamma \leq \gamma_{0}$.

Furthermore, with this choice of $\eta$, we have

$$
\lfloor\eta B\rfloor /\lfloor K \kappa\rfloor \geq C_{-} /\left(8 C_{+}\right)=: \eta_{1},
$$

uniformly in the same range of $\rho, \gamma$ and $s$, provided that $\lfloor\eta B\rfloor \geq 1$; hence, again from Lemma 1, taking $\alpha=\alpha\left(\eta_{1}\right)$, we have

$$
\sum_{m<B-\lfloor\eta B\rfloor} \pi^{0}\{m+A\}=\pi^{0}[1,\lfloor K \kappa\rfloor-\lfloor\eta B\rfloor-1]=O\left(K^{-1 / 2}\right)
$$

and

$$
\sum_{m>B+\lfloor\eta B\rfloor} \pi^{0}\{m+A\}=\sum_{j>\lfloor K \kappa\rfloor+\lfloor\eta B\rfloor} \pi^{0}\{j\}=O\left(K^{-1 / 2}\right) ;
$$

the theorem now follows from (2.18), (2.19) and (2.26)-(2.29).

Remark 1. The shifted Poisson approximation of Theorem 1 is as simple to understand as the more usual normal approximation, but is much stronger, being expressed in terms of the total variation metric. Despite this, the order $O\left(K^{-1 / 2}\right)$ of the error bound is as good as the best that could ever be attained using a normal approximation, since then the discretization error alone would have to be of this order; the discrete distribution being approximated has point probabilities of order $O\left(K^{-1 / 2}\right)$, whereas the normal distribution is continuous on $\mathbb{R}$. The choice of shifted Poisson is found by matching its mean and variance to those of $\pi^{0}$, asymptotically as $K \rightarrow \infty$. Note that any match has to take into account that the shift can only be by an integer amount, because the resulting distribution must take values in the integers.

Having established the detailed form of $\pi^{0(s)}$ for any $0 \leq s \leq 2 K$, we now need to show that, indeed, the equilibrium distribution $\pi^{(s)}$ of $Z^{(s)}$ is close to the distribution $\pi^{(s)}\{0\} \Delta_{\{0\}}+\left(1-\pi^{(s)}\{0\}\right) \pi^{0(s)}$. In order to do this, we need bounds for some absorption probabilities and mean recurrence times associated with the process $Z^{(s)}$, again uniformly in $\rho \leq 1, \gamma \leq \gamma_{0}$ and $s \leq 2 K$, and now, additionally, in $v \geq 0$. Once more, we suppress the dependence on $s$ and $K$, and let $\tau^{\{0\}}$ denote the time of first hitting the state $0, \tau^{\{0,\lfloor K \kappa\rfloor\}}$ the time of first hitting the set $\{0,\lfloor K \kappa\rfloor\}$. 
Lemma 2. Under Conditions 1-5,

$$
\mathbb{E}^{j} \tau^{\{0,\lfloor K \kappa\rfloor\}}=O\left(v^{-1} \wedge \log (1+|j-\lfloor K \kappa\rfloor|) \text { for all } j \geq 1 .\right.
$$

Proof. Writing $e_{j}:=\mathbb{E}^{j} \tau^{\{0,\lfloor K \kappa\rfloor\}}$, it is immediate that $e_{j} \leq v^{-1}$. Observe also that $\left(e_{j}\right)_{j \geq 0}$ is the finite solution to the equations

$$
\left(\lambda_{j}+\mu_{j}+v\right) e_{j}=1+\lambda_{j} e_{j+1}+\mu_{j} e_{j-1}, \quad j \geq 1, \quad j \neq\lfloor K \kappa\rfloor,
$$

which also satisfies $e_{0}=e_{\lfloor K \kappa\rfloor}=0$. In $j>\lfloor K \kappa\rfloor$, we write these equations as

$$
e_{j}-e_{j-1}=\frac{\lambda_{j}}{\mu_{j}}\left(e_{j+1}-e_{j}\right)+\frac{1-v e_{j}}{\mu_{j}}, \quad j>\lfloor K \kappa\rfloor,
$$

where $0 \leq 1-v e_{j} \leq 1$, since $v e_{j}=0$ if $v=0$ and $e_{j}^{-1} \leq v^{-1}$ otherwise, entailing

$$
e_{j}-e_{j-1} \leq \sum_{r \geq 0} \frac{1}{\mu_{j+r}} \prod_{l=0}^{r-1} \frac{\lambda_{j+l}}{\mu_{j+l}} \leq \frac{1}{\mu_{j}-\lambda_{j}}, \quad j \geq\lfloor K \kappa\rfloor+1 .
$$

Thus, by (2.6) and because $e_{j}=\sum_{l=\lfloor K \kappa\rfloor+1}^{j}\left(e_{l}-e_{l-1}\right)$, the claim is proved in the case $j>\lfloor K \kappa\rfloor$.

For $1 \leq j<\lfloor K \kappa\rfloor$, we rewrite (2.30) as

$$
e_{j}-e_{j+1}=\frac{\mu_{j}}{\lambda_{j}}\left(e_{j-1}-e_{j}\right)+\frac{1-v e_{j}}{\lambda_{j}}, \quad 1 \leq j<\lfloor K \kappa\rfloor,
$$

together with the side conditions

$$
\sum_{j=0}^{\lfloor K \kappa\rfloor-1}\left(e_{j}-e_{j+1}\right)=e_{0}-e_{\lfloor K \kappa\rfloor}=0 ;
$$

this in particular yields

$$
\begin{aligned}
e_{j}-e_{j+1} & =\sum_{r=0}^{j-1}\left(\frac{1-v e_{j-r}}{\lambda_{j-r}}\right) \prod_{l=1}^{r} \frac{\mu_{j-l+1}}{\lambda_{j-l+1}}-e_{1} \prod_{l=1}^{j} \frac{\mu_{j-l+1}}{\lambda_{j-l+1}} \\
& \leq \sum_{r=0}^{j-1}\left(\frac{1}{\lambda_{j-r}}\right) \prod_{l=1}^{r} \frac{\mu_{j-l+1}}{\lambda_{j-l+1}}, \quad 1 \leq j<\lfloor K \kappa\rfloor .
\end{aligned}
$$

Now, since $\mu_{j} / \lambda_{j}$ is non-decreasing in $j$, the bound in (2.32) is at most

$$
\frac{1}{\lambda_{j}} \sum_{r=0}^{j-1} \frac{j}{j-r}\left(\frac{\mu_{j}}{\lambda_{j}}\right)^{r} \leq \frac{1}{\lambda_{j}}\left(\frac{\lambda_{j}}{\lambda_{j}-\mu_{j}}\right)^{2} .
$$

Hence, for $j \leq\lfloor K \kappa / 2\rfloor$, we have $\mu_{j} / \lambda_{j} \leq\left\{d(1 / 2)+\gamma_{0}\right\} / b(1 / 2)=c_{0}<1$ by Condition $5 \mathrm{~d}$, and thus

$$
e_{j}-e_{j+1} \leq \frac{1}{\lambda_{j}\left(1-c_{0}\right)^{2}}, \quad j \leq\lfloor K \kappa / 2\rfloor ;
$$


for $\lfloor K \kappa / 2\rfloor<j<\lfloor K \kappa\rfloor$, we use (2.32) to give the bound

$$
\begin{aligned}
e_{j}-e_{j+1} & \leq \frac{1}{\lambda_{\lfloor K \kappa / 2\rfloor}}\left(\frac{\lambda_{j}}{\lambda_{j}-\mu_{j}}\right)+\frac{1}{\lambda_{\lfloor K \kappa / 2\rfloor}\left(1-c_{0}\right)^{2}}\left(\frac{\mu_{j}}{\lambda_{j}}\right)^{j-\lfloor K \kappa\rfloor} \\
& \leq \frac{1}{\lambda_{\lfloor K \kappa / 2\rfloor}}\left(\frac{\lambda_{j}}{\lambda_{j}-\mu_{j}}+\frac{1}{\left(1-c_{0}\right)^{2}}\right) .
\end{aligned}
$$

Invoking (2.5), and because $e_{j}=\sum_{l=j}^{\lfloor K \kappa\rfloor-1}\left(e_{l}-e_{l-1}\right)$, the proof is complete.

In the next Lemma, where again all order statements are uniform in $\rho \leq 1$, $\gamma \leq \gamma_{0}, s \leq 2 K$ and $v \geq 0$, we consider

$$
p_{0}(j ;\lfloor K \kappa\rfloor)=p_{0}^{(s)}(j ;\lfloor K \kappa\rfloor):=\mathbb{P}^{j}\left[Z^{(s)}\left(\tau^{\{0,\lfloor K \kappa\rfloor\}}\right)=0\right] .
$$

Lemma 3. Defining $c_{j j}:=\prod_{l=1}^{j-1} \frac{\mu_{l}}{\lambda_{l}}$, we have

$$
1-p_{0}(1 ;\lfloor K \kappa\rfloor)=\left(\sum_{j=1}^{\lfloor K \kappa\rfloor} c_{j j}\right)^{-1}\{1+O(v \log K)\} .
$$

If $\lfloor K \kappa / 2\rfloor \leq j<\lfloor K \kappa\rfloor$, then

$$
p_{0}(j ;\lfloor K \kappa\rfloor)=O\left(v \log (1+|\lfloor K \kappa\rfloor-j|)+K e^{-\alpha_{0} K}\right)
$$

for $\alpha_{0}$ such that $e^{-\alpha_{0}}=c_{0}^{3 / 8}$, where $c_{0}$ is as defined in Condition $5 d$.

If $j>\lfloor K \kappa\rfloor$, then

$$
p_{0}(j ;\lfloor K \kappa\rfloor)=O(v \log (1+|\lfloor K \kappa\rfloor-j|)) .
$$

Proof. Writing $p_{j}$ for $p_{0}(j ;\lfloor K \kappa\rfloor)$, observe that $\left(p_{j}\right)_{j \geq 0}$ satisfy the equations

$$
\left(\lambda_{j}+\mu_{j}+v\right) p_{j}=\lambda_{j} p_{j+1}+\mu_{j} p_{j-1}+v, \quad j \geq 1,
$$

with the side conditions $p_{0}=1, p_{\lfloor K \kappa\rfloor}=0$. Now (2.34) gives

$$
\begin{aligned}
p_{j}-p_{j+1} & =\lambda_{j}^{-1}\left\{\mu_{j}\left(p_{j-1}-p_{j}\right)+v\left(1-p_{j}\right)\right\} \\
& =v \sum_{r=0}^{j-1} \frac{1-p_{j-r}}{\lambda_{j-r}} \prod_{l=0}^{r-1} \frac{\mu_{j-l}}{\lambda_{j-l}}+\left(1-p_{1}\right) \prod_{l=0}^{j-1} \frac{\mu_{j-l}}{\lambda_{j-l}} .
\end{aligned}
$$

Adding (2.35) over $1 \leq j \leq\lfloor K \kappa\rfloor-1$ and adding $\left(1-p_{1}\right)$ gives

$$
1=\left(1-p_{1}\right) \sum_{j=1}^{\lfloor K \kappa\rfloor} c_{j j}+v \eta
$$

where

$$
0 \leq \eta \leq \sum_{j=1}^{\lfloor K \kappa\rfloor-1} \sum_{r=0}^{j-1}\left(\frac{1}{\lambda_{j-r}}\right) \prod_{l=1}^{r} \frac{\mu_{j-l+1}}{\lambda_{j-l+1}}=O(\log K)
$$


since the inner sum may be bounded as in the previous lemma. This proves the first part of the lemma.

Note also that

$$
c_{j j} \leq c_{0}^{(j-1) \wedge\lfloor K \kappa / 2\rfloor},
$$

so that

$$
\begin{aligned}
1 & <\sum_{j=1}^{\lfloor K \kappa\rfloor} c_{j j}<\left(1-c_{0}\right)^{-1}+(\lfloor K \kappa / 2\rfloor+1) c_{0}^{\lfloor K \kappa / 2\rfloor} \\
& <\left(1-c_{0}\right)^{-1}+1+\left\{\log \left(1 / c_{0}\right)\right\}^{-1}=: 1 /(2 \bar{p})<\infty .
\end{aligned}
$$

Hence it follows that

$$
1-p_{0}(1 ;\lfloor K \kappa\rfloor) \geq \bar{p}>0,
$$

uniformly in $v \log K \leq \eta_{0}$, for suitable choice of $\eta_{0}>0$; this lower bound is also uniform in $\rho \leq 1, \gamma \leq \gamma_{0}$ and $s \geq 0$, in the above range of $\nu$.

For the second part, add (2.35) over $J \leq j \leq\lfloor K \kappa\rfloor-1$ to give

$$
\begin{aligned}
p_{J} & =\left(1-p_{1}\right) \sum_{j=J+1}^{\lfloor K \kappa\rfloor} c_{j j}+O(v \log (1+|\lfloor K \kappa\rfloor-J|)) \\
& =O\left(|\lfloor K \kappa\rfloor-J| c_{0}^{\lfloor K \kappa / 2\rfloor}+v \log (1+|\lfloor K \kappa\rfloor-J|)\right)
\end{aligned}
$$

for all $\lfloor K \kappa / 2\rfloor \leq J \leq\lfloor K \kappa\rfloor-1$, by (2.37), completing the proof of the second part for such $J$.

If $j>\lfloor K \kappa\rfloor$, the proof is similar:

$$
\begin{aligned}
p_{j}-p_{j-1} & =\frac{\lambda_{j}}{\mu_{j}}\left(p_{j+1}-p_{j}\right)+\frac{v}{\mu_{j}}\left(1-p_{j}\right) \\
& \leq v \sum_{r \geq 0} \frac{1}{\mu_{j+r}} \prod_{l=1}^{r} \frac{\lambda_{j+l-1}}{\mu_{j+l-1}} \\
& \leq v /\left(\mu_{j}-\lambda_{j}\right),
\end{aligned}
$$

which is enough.

With these preparatory results, we can turn to the proof of the main approximation to the equilibrium distribution of $Z^{(s)}$.

Theorem 2. Let $\pi:=\pi^{(s)}$ denote the equilibrium distribution of $Z^{(s)}$. Then, under Conditions 1-5,

$$
\pi\{0\}=\left\{1+\rho \gamma s E_{K}\left(1-p_{0}(1 ;\lfloor K \kappa\rfloor)\right)\right\}^{-1}\left\{1+O\left(\varepsilon_{0}(K, \nu)\right)\right\},
$$

uniformly in $0 \leq s \leq 2 K, \rho \leq 1, \gamma \leq \gamma_{0}$ and $v \log K \leq \eta_{0}$, where

$$
E_{K}:=E_{K}^{(s)}:=\mathbb{E}^{\lfloor K \kappa\rfloor} \tau^{\{0\}} \text { and } \varepsilon_{0}(K, v):=E_{K}^{-1} \log K,
$$


and where $\eta_{0}$ is as in (2.39). Furthermore, if

$$
\pi^{\prime}:=\pi\{0\} \Delta_{\{0\}}+(1-\pi\{0\}) \pi^{0},
$$

where $\pi^{0}$ is as defined in (2.3), then $d_{T V}\left(\pi, \pi^{\prime}\right)=O\left(\varepsilon_{1}(K, v)\right)$, with

$$
\varepsilon_{1}(K, v):=\varepsilon_{0}(K, v)+K e^{-\alpha_{1} K},
$$

and $\alpha_{1}:=\min \left(\alpha_{0}, \alpha(1 / 2)\right)$, the latter defined in Lemmas 1 and (3). Finally,

$$
G(s)=K \kappa\left(1-\pi^{(s)}\{0\}\right)\left\{1+O\left(\varepsilon_{2}(K, v)\right)\right\},
$$

where $\varepsilon_{2}(K, v):=\varepsilon_{0}(K, v)+K^{-1 / 2}$.

Proof. If $\rho \gamma s=0$, then $\pi=\Delta_{\{0\}}$ is degenerate at 0 , and all the statements of the theorem are immediate; in what follows, we therefore assume that $\rho \gamma s>0$.

For any bounded $f: \mathbf{Z}_{+} \rightarrow \mathbb{R}$, define $\theta(f)$ by

$$
\theta(f)(j):=-\int_{0}^{\infty}\left\{\mathbb{E}^{j} f\left(Z_{t}\right)-\pi(f)\right\} d t, \quad j \geq 0 .
$$

Taking $j=0$, it follows that

$$
\begin{aligned}
\theta(f)(0) & =-\mathbb{E}^{0}\left(\tau^{(1)}\right)(f(0)-\pi(f))+\theta(f)(1) \\
& =-(1 / \rho \gamma s)(f(0)-\pi(f))+\theta(f)(1),
\end{aligned}
$$

where $\tau^{(1)}$ denotes the time of first hitting the state 1 ; thus

$$
\rho \gamma s\{\theta(f)(1)-\theta(f)(0)\}=f(0)-\pi(f) .
$$

Next, taking $j \geq 1$, it follows that

$$
\begin{gathered}
\theta(f)(j)=-\mathbb{E}^{j} \int_{0}^{\tau^{\{0,\lfloor K \kappa\rfloor\}}}\left(f\left(Z_{t}\right)-\pi(f)\right) d t+p_{0}(j ;\lfloor K \kappa\rfloor) \theta(f)(0) \\
+\left(1-p_{0}(j ;\lfloor K \kappa\rfloor)\right) \theta(f)(\lfloor K \kappa\rfloor) .
\end{gathered}
$$

For $j=1,(2.42)$ gives

$$
\begin{aligned}
& \left|\{\theta(f)(\lfloor K \kappa\rfloor)-\theta(f)(0)\}\left(1-p_{0}(1 ;\lfloor K \kappa\rfloor)\right)-\{\theta(f)(1)-\theta(f)(0)\}\right| \\
& \leq \max _{1 \leq j<\lfloor K \kappa\rfloor}|f(j)-\pi(f)| \mathbb{E}^{1} \tau^{\{0,\lfloor K \kappa\rfloor\}}=O\left(\log K\|f\|_{d}\right),
\end{aligned}
$$

by Lemma 2, where $\|f\|_{d}:=\sup _{j, j^{\prime}}\left|f(j)-f\left(j^{\prime}\right)\right|$; for $f=1_{\{0\}}$, and using (2.41), this strengthens to

$$
\begin{gathered}
\left|\left\{\theta\left(1_{\{0\}}\right)(\lfloor K \kappa\rfloor)-\theta\left(1_{\{0\}}\right)(0)\right\}\left(1-p_{0}(1 ;\lfloor K \kappa\rfloor)\right)-(1-\pi\{0\}) /(\rho \gamma s)\right| \\
\leq \pi\{0\} \mathbb{E}^{1} \tau^{\{0,\lfloor K \kappa\rfloor\}}=O(\pi\{0\} \log K) .
\end{gathered}
$$

The first statement of the theorem is now proved, by what is essentially a renewal argument, using (2.44) together with an alternative expression for the difference $\theta\left(1_{\{0\}}\right)(\lfloor K \kappa\rfloor)-\theta\left(1_{\{0\}}\right)(0)$, which is derived from (2.40) by writing

$$
\theta(f)(\lfloor K \kappa\rfloor)=-\mathbb{E}^{\lfloor K \kappa\rfloor} \int_{0}^{\tau^{\{0\}}}\left(f\left(Z_{t}\right)-\pi(f)\right) d t+\theta(f)(0),
$$


with $f=1_{\{0\}}$. This, with (2.41), gives

$$
\left(1-p_{0}(1 ;\lfloor K \kappa\rfloor)\right)^{-1}(\{1-\pi\{0\}\} /(\rho \gamma s)+O(\log K) \pi\{0\})=\pi\{0\} \mathbb{E}^{\lfloor K \kappa\rfloor} \tau^{\{0\}}
$$

so that

$$
(1-\pi\{0\}) / \pi\{0\}=\rho \gamma s E_{K}\left(1-p_{0}(1 ;\lfloor K \kappa\rfloor)\right)\left\{1+O\left(\varepsilon_{0}(K, v)\right)\right\} .
$$

This establishes the first part of the theorem.

For the second part, fix any bounded $f$, and note that the function $h:=\theta(f)$ satisfies the equation $\mathcal{A} h=f-\pi(f)$, as shown in the proof of [3], Theorem 2.4. Taking expectations with respect to the probability measure $\pi^{\prime}$ thus gives

$$
\begin{aligned}
& \pi^{\prime}(f)-\pi(f)=\pi^{\prime}(\mathcal{A} h)=\pi\{0\} \rho \gamma s\{h(1)-h(0)\}+(1-\pi\{0\}) \\
& \quad \times \sum_{j \geq 1} \pi^{0}\{j\}\left\{\lambda_{j}(h(j+1)-h(j))+\mu_{j}(h(j-1)-h(j))+v(h(0)-h(j))\right\} .
\end{aligned}
$$

Now, for $j \geq 2$, the coefficient of $h(j)$ in (2.47) is

$$
\lambda_{j-1} \pi^{0}\{j-1\}-\left(\lambda_{j}+\mu_{j}\right) \pi^{0}\{j\}+\mu_{j+1} \pi^{0}\{j+1\}-v=-v,
$$

by the definition of $\pi^{0}$, leaving

$$
\begin{gathered}
\pi^{\prime}(f)-\pi(f)=\pi\{0\} \rho \gamma s\{h(1)-h(0)\}-(1-\pi\{0\})\{h(1)-h(0)\} \mu_{1} \pi^{0}\{1\} \\
-(1-\pi\{0\}) \nu \sum_{j \geq 1} \pi^{0}\{j\}\{h(j)-h(0)\} .
\end{gathered}
$$

Now, from (2.42), for $\lfloor K \kappa / 2\rfloor \leq j \leq\lfloor 2 K \kappa\rfloor$, we have

$$
|h(\lfloor K \kappa\rfloor)-h(j)| \leq p_{0}(j ;\lfloor K \kappa\rfloor)|h(\lfloor K \kappa\rfloor)-h(0)|+\mathbb{E}^{j} \tau^{\{0,\lfloor K \kappa\rfloor\}}\|f\|_{d},
$$

and $|h(j)-h(0)| \leq v^{-1}\|f\|_{d}$ for all $j$, from the definition of $h$ in (2.40) and a coupling of two $Z^{(s)}$-processes, one starting in $j$ and one in equilibrium, by matching them at the first catastrophe. Hence it follows that

$$
\begin{aligned}
v|h(\lfloor K \kappa\rfloor)-h(j)| & =O\left(p_{0}(j ;\lfloor K \kappa\rfloor)+v \mathbb{E}^{j} \tau^{\{0,\lfloor K \kappa\rfloor\}}\right)\|f\|_{d} \\
& =O\left(v \log K+K e^{-\alpha_{0} K}\right)\|f\|_{d},
\end{aligned}
$$

from Lemmas 2 and (3). Invoking (2.43), we thus have

$$
\begin{gathered}
(1-\pi\{0\}) \nu\left|\{h(j)-h(0)\}-\left(1-p_{0}(1 ;\lfloor K \kappa\rfloor)\right)^{-1}\{h(1)-h(0)\}\right| \\
=O\left(v \log K+K e^{-\alpha_{0} K}\right)\|f\|_{d},
\end{gathered}
$$

for all $\lfloor K \kappa / 2\rfloor \leq j \leq\lfloor 2 K \kappa\rfloor$, uniformly in $\nu \log K \leq \eta_{0}$. Also, once again since $|h(j)-h(0)| \leq v^{-1}\|f\|_{d}$ for all $j$, it follows that

$$
\begin{aligned}
& (1-\pi\{0\}) \nu\left(\sum_{j=1}^{\lfloor K \kappa / 2\rfloor-1}+\sum_{j \geq\lfloor 2 K \kappa\rfloor+1}\right) \pi^{0}\{j\}|h(j)-h(1)| \\
& =O\left(K^{-1 / 2} e^{-\alpha(1 / 2) K}\right)\|f\|_{d},
\end{aligned}
$$


from Lemma 1. Combining these estimates with (2.48) thus yields

$$
\begin{aligned}
& \pi^{\prime}(f)-\pi(f)=\pi\{0\} \rho \gamma s\{h(1)-h(0)\} \\
& \quad-\frac{(1-\pi\{0\})\left\{v+\mu_{1} \pi^{0}\{1\}\left(1-p_{0}(1 ;\lfloor K \kappa\rfloor)\right)\right\}}{\left(1-p_{0}(1 ;\lfloor K \kappa\rfloor)\right)}\{h(1)-h(0)\} \\
& +O\left(\varepsilon_{1}(K, v)\right)\|f\|_{d} .
\end{aligned}
$$

However, from (2.49) and (2.41), taking $f=1_{\{0\}}$, it follows that

$$
\begin{aligned}
0=(1- & \pi\{0\})\left\{\pi\{0\}-\frac{1}{\rho \gamma s}\left(\frac{(1-\pi\{0\})\left\{\nu+\mu_{1} \pi^{0}\{1\}\left(1-p_{0}(1 ;\lfloor K \kappa\rfloor)\right)\right\}}{\left(1-p_{0}(1 ;\lfloor K \kappa\rfloor)\right)}\right)\right\} \\
& +O\left(\varepsilon_{1}(K, \nu)\right) ;
\end{aligned}
$$

this, combined with (2.49) and because

$$
\rho \gamma \sigma\{h(1)-h(0)\} /(1-\pi\{0\}) \leq\|f\|_{d},
$$

gives

$$
\left|\pi^{\prime}(f)-\pi(f)\right|=O\left(\varepsilon_{1}(K, v)\right)\|f\|_{d},
$$

completing the second part of the proof.

For the last part, write

$G(s)=\sum_{j \geq 1} j \pi\{j\}=\left\{\sum_{j=1}^{\lfloor 2 K \kappa\rfloor} j \pi^{\prime}\{j\}+\sum_{j=1}^{\lfloor 2 K \kappa\rfloor} j\left(\pi\{j\}-\pi^{\prime}\{j\}\right)\right\}+\sum_{j>\lfloor 2 K \kappa\rfloor} j \pi\{j\}$.

The total variation approximation (2.51) just established shows that

$$
K^{-1} \sum_{j=1}^{\lfloor 2 K \kappa\rfloor} j\left|\pi\{j\}-\pi^{\prime}\{j\}\right|=O\left(\varepsilon_{1}(K, v)\right),
$$

and

$$
K^{-1} \sum_{j=1}^{\lfloor 2 K \kappa\rfloor} j \pi^{0}\{j\}=\kappa\left\{1+O\left(K^{-1 / 2}\right)\right\}
$$

from parts 1,2 and 4 of Lemma 1 , which, with $\pi^{\prime}\{j\}=(1-\pi\{0\}) \pi^{0}\{j\}$, establishes the main contribution to $G(s)$. Finally, a simple coupling argument shows that $\sum_{j>\lfloor 2 K \kappa\rfloor} j \pi\{j\} \leq \sum_{j>\lfloor 2 K \kappa\rfloor} j \pi^{0}\{j\}$, so that, by Lemma 1.3,

$$
K^{-1} \sum_{j>\lfloor 2 K \kappa\rfloor} j \pi\{j\}=O\left(e^{-\alpha K}\right)
$$

for some $\alpha>0$. This completes the proof of the theorem. 
Remark 2. Combining (2.50) and the formula for $\pi^{(s)}\{0\}$, it follows that

$$
1 / E_{K}^{(s)}=\left(v+\mu_{1} \pi^{0(s)}\{1\}\left(1-p_{0}(1 ;\lfloor K \kappa\rfloor)\right)\right)\left\{1+O\left(\varepsilon_{1}(K, v)\right)\right\} .
$$

Now $\mu_{1} \pi^{0(s)}\{1\}=O\left(K^{1 / 2} e^{-\alpha(1 / 2) K}\right)$ from Lemma 1 ; hence $E_{K}^{(s)}$ can mostly be replaced by $v^{-1}$ in the formulae. Note also that, in any case, $E_{K}^{(s)} \leq v^{-1}$.

Provided that $\varepsilon_{2}(K, v)$ is small enough, Theorem 2 implies a corresponding result for the non-degenerate equilibrium solution of the differential equations (1.2), which is the equilibrium distribution $\pi^{\left(s^{*}\right)}$ corresponding to that value $s^{*}>0$ of $s$ which solves the equation $s=G(s)$.

Corollary 1. Suppose that Conditions 1-5 hold, and that $G^{\prime}(0)>1$. Let $s^{*}$ be the positive solution of the equation $G(s)=s$. Then there exist $K_{1}, \eta_{1}>0$ such that, uniformly in $\rho \leq 1, \gamma \leq \gamma_{0}, K \geq K_{1}$ and $v \leq \eta_{1} / \log K$, the non-trivial equilibrium distribution $\pi:=\pi_{e n}:=\pi^{\left(s^{*}\right)}$ to (1.2) satisfies

(1) $\pi\{0\}=\left\{\lfloor K \kappa\rfloor \rho \gamma E_{K}\left(1-p_{0}(1 ;\lfloor K \kappa\rfloor)\right)\right\}^{-1}\left\{1+O\left(\varepsilon_{2}(K, v)\right)\right\}$;

(2) $d_{T V}\left(\pi, \pi^{\prime}\right)=O\left(\varepsilon_{1}(K, v)\right)$;

(3) $d_{T V}\left(\pi, \pi^{\prime \prime}\right)=O\left(\varepsilon_{2}(K, v)\right)$.

Here

$$
\pi^{\prime}:=\pi\{0\} \Delta_{\{0\}}+(1-\pi\{0\}) \pi^{0},
$$

with $\pi^{0}$ given by (2.3), and

$$
\pi^{\prime \prime}:=\pi\{0\} \Delta_{\{0\}}+(1-\pi\{0\})\left(\Delta_{\lfloor K \kappa\rfloor} * \widehat{\mathrm{Po}}(B)\right),
$$

with $B$ as in Theorem 1. Throughout, the quantities $\pi, \pi^{0}, \kappa, E_{K}, p_{0}$ and $B$ are to be understood with $s=s^{*}$.

Proof. The corollary follows from Theorems 2 and 1, so long as $s^{*} \leq 2 K$ and $\eta_{1} \leq \eta_{0}$, because then

$$
s^{*}=G\left(s^{*}\right)=K \kappa\left(1-\pi^{\left(s^{*}\right)}\{0\}\right)\left\{1+O\left(\varepsilon_{2}(K, v)\right)\right\} .
$$

But, from (2.4), we have $\kappa^{(s)} \leq 5 / 4$ for all $0 \leq s \leq 2 K$, and it thus follows from Theorem 2 that $G(s) \leq 2 K$ for all $0 \leq s \leq 2 K$, and hence that $s^{*} \leq 2 K$, whenever $\varepsilon_{2}(K, v)$ is small enough. The conditions of the corollary relating to $v$ and $K$ are chosen to ensure this.

Remark 3. The parallel with Levins's theorem is now apparent. If Conditions 1-5 hold, and if $K$ is large enough and $v \log K$ small enough, then there is a proportion $\pi\{0\}$ of empty patches, and the remaining patches have populations of comparable sizes $K \kappa+O\left(K^{1 / 2}\right)$. The rôle of Levins's extinction rate $v_{L}$ is played by $E_{K}^{-1}$, incorporating extinction both through catastrophe and through demographic fluctuation, and is close to the catastrophe rate $v$ so long as $v^{-1} K e^{-\alpha_{1} K}$ is small. The analogue of Levins's colonization rate $c_{L}$ is thus $\rho \gamma K \kappa\left(1-p_{0}(1 ;\lfloor K \kappa\rfloor)\right)$, by the formula for $\pi\{0\}$; the product $\gamma K \kappa$ represents the rate at which migrants leave an occupied patch, a proportion $\rho$ of these survive to reach a new patch, and a proportion $p_{0}(1 ;\lfloor K \kappa\rfloor)$ of migrations into empty patches then fail to result in subsequent colonization, because of extinction occurring by chance before the population becomes established. 


\section{Approximations to the equilibrium colonization rate}

We now establish approximations to the solution $s^{*}$ of the fixed point equation (1.6) under Conditions 1-5. Since the dependence on $s$ is important here, we shall carry it in the notation, but, now and in all that follows, by way of the variable $\sigma=s / K$, $0 \leq \sigma \leq 2$; as before, the $K$-dependence is suppressed, and order terms are to be understood as being uniform in $\rho \leq 1$ and $\gamma \leq \gamma_{0}$. Except in the next lemma, we also assume that $v \log K \leq \eta_{0}$.

We begin by recalling that $\kappa^{(\sigma)}$ is the solution to the equation

$$
\kappa b(\kappa)+\rho \gamma \sigma=\kappa(d(\kappa)+\gamma),
$$

so that $\kappa^{(\sigma)}$ is increasing in $\sigma$; from Conditions $5 \mathrm{a}$ and $5 \mathrm{~b}$, it thus follows that

$$
3 / 4<x_{-}=\kappa^{(0)} \leq \kappa^{(\sigma)} \leq \kappa^{(2)}=x_{+}<5 / 4 .
$$

It also follows from (3.1) and (2.25) that

$$
\frac{d \kappa^{(\sigma)}}{d \sigma}=\frac{\gamma}{\kappa\left(d^{\prime}(\kappa)-b^{\prime}(\kappa)\right)+\rho \gamma \sigma / \kappa} \leq \frac{\gamma}{x_{-}\left(d^{\prime}\left(x_{-}\right)-b^{\prime}\left(x_{-}\right)\right)}<1 / 2,
$$

this last by Condition 5e.

The first Lemma concerns the approximation of $p_{0}^{(\sigma)}\left(1 ;\left\lfloor K \kappa^{(\sigma)}\right\rfloor\right)$, defined in (2.33), with the simpler decreasing function $p_{0}^{(\sigma)}$ defined by

$$
\left(1-p_{0}^{(\sigma)}\right)^{-1}:=\sum_{j \geq 0} \zeta^{j} \prod_{l=1}^{j}\left(1+\frac{K \rho \gamma \sigma}{b(0) l}\right)^{-1}
$$

where

$$
0<\zeta:=(d(0)+\gamma) / b(0) \leq\left(d(0)+\gamma_{0}\right) / b(0)=: c_{2}<1
$$

Note that $0 \leq p_{0}^{(\sigma)} \leq \zeta$

Lemma 4. We have

$$
\left|p_{0}^{(\sigma)}-p_{0}^{(\sigma)}\left(1 ;\left\lfloor K \kappa^{(\sigma)}\right\rfloor\right)\right|=O\left(K^{-1}\right)+O(v \log K),
$$

uniformly in $0 \leq \sigma \leq 2$ and in $v \geq 0$.

Proof. In the following argument, we make frequent use of (2.4), together with the properties of the functions $b$ and $d$, and the fact that $b(x)+\rho \gamma \sigma / x>d(x)+\gamma$ for all $x<\kappa^{(\sigma)}$.

By Lemma 3, we can write

$$
\left(1-p_{0}^{(\sigma)}\left(1 ;\left\lfloor K \kappa^{(\sigma)}\right\rfloor\right)\right)^{-1}=\sum_{j=1}^{\left\lfloor K \kappa^{(\sigma)}\right\rfloor j-1} \prod_{l=1}^{j-1} a_{l}\{1+O(v \log K)\},
$$


while, by definition,

$$
\left(1-p_{0}^{(\sigma)}\right)^{-1}=\sum_{j=1}^{\infty} \prod_{l=1}^{j-1} a_{l}^{\prime}
$$

where

$$
a_{l}^{\prime}=\frac{d(0)+\gamma}{b(0)+K \rho \gamma \sigma l^{-1}}<\frac{d(l / K)+\gamma}{b(l / K)+K \rho \gamma \sigma l^{-1}}=a_{l} .
$$

It is easy to see that $a_{l}$ and $a_{l}^{\prime}$ are increasing with $l$, and satisfy

$$
a_{l}-a_{l}^{\prime} \leq \frac{l M}{K}
$$

where

$$
M:=b(0)^{-1}\left\{\sup _{0 \leq x \leq 5 / 4} d^{\prime}(x)+\sup _{0 \leq x \leq 5 / 4}\left|b^{\prime}(x)\right|\right\}
$$

furthermore,

$$
a_{\left\lfloor K \kappa^{(\sigma)}\right\rfloor} \leq \frac{d\left(\kappa^{(\sigma)}\right)+\gamma}{b\left(\kappa^{(\sigma)}\right)+\rho \gamma \sigma / \kappa^{(\sigma)}}=1 .
$$

We now compute

$$
\begin{aligned}
& \sum_{j=1}^{\lfloor K / 2\rfloor}\left(\prod_{l=1}^{j-1} a_{l}-\prod_{l=1}^{j-1} a_{l}^{\prime}\right)=\sum_{j=2}^{\lfloor K / 2\rfloor} \sum_{m=1}^{j-1}\left(a_{m}-a_{m}^{\prime}\right) \prod_{l=1}^{m-1} a_{l} \prod_{k=m+1}^{j-1} a_{k}^{\prime} \\
& \leq \sum_{j=2}^{\lfloor K / 2\rfloor} \sum_{m=1}^{j-1}\left(a_{m}-a_{m}^{\prime}\right) \prod_{\substack{l=1 \\
l \neq m}}^{j-1} a_{l}=\sum_{m=1}^{\lfloor K / 2\rfloor-1}\left(a_{m}-a_{m}^{\prime}\right) \sum_{j=m+1}^{\lfloor K / 2\rfloor} \prod_{\substack{l=1 \\
l \neq m}}^{j-1} a_{l} \\
& \leq \sum_{m=1}^{\lfloor K / 2\rfloor-1}\left(a_{m}-a_{m}^{\prime}\right) \sum_{j=m+1}^{\lfloor K / 2\rfloor} c_{0}^{j-2} \leq \frac{1}{1-c_{0}} \sum_{m=1}^{\lfloor K / 2\rfloor-1}\left(a_{m}-a_{m}^{\prime}\right) c_{0}^{m-1} \\
& \leq \frac{M}{K}\left(1-c_{0}\right)^{-3},
\end{aligned}
$$

using (3.9), where

$$
0<c_{0}=\frac{d(1 / 2)+\gamma_{0}}{b(1 / 2)}<1,
$$

by Condition 5d. Moreover, we also have

$$
\sum_{j=\lfloor K / 2\rfloor+1}^{\left\lfloor K \kappa^{(\sigma)}\right\rfloor} \prod_{l=1}^{j-1} a_{l} \leq c_{0}^{K / 2-2} K\left(\kappa^{(\sigma)}-\eta\right) \leq(5 K / 4) c_{0}^{K / 2-2},
$$


while

$$
\sum_{j=\lfloor K / 2\rfloor+1}^{\infty} \prod_{l=1}^{j-1} a_{l}^{\prime} \leq \frac{\zeta^{K / 2-1}}{1-\zeta}
$$

Using (3.10), (3.11) and (3.12) together with (3.7) and (3.8), and because $K^{2} c_{0}^{K}$ is bounded, we now see that

$$
\left|\left(1-p_{0}^{(\sigma)}\left(1 ;\left\lfloor K \kappa^{(\sigma)}\right\rfloor\right)\right)^{-1}-\left(1-p_{0}^{(\sigma)}\right)^{-1}\right|=O\left(K^{-1}\right)+O(\nu \log K),
$$

and the lemma follows.

Now, using Lemma 4 together with (2.46) and (2.52), we have

$$
\left(1-\pi^{(\sigma)}\{0\}\right)=\frac{\sigma g(\sigma)}{1+\sigma g(\sigma)}\left\{1+O\left(\varepsilon_{0}(K, v)+K^{-1}+v^{-1} K^{1 / 2} e^{-\alpha_{1} K}\right)\right\},
$$

where

$$
g(\sigma):=K \rho \gamma v^{-1}\left(1-p_{0}^{(\sigma)}\right),
$$

and hence, from Theorem 2,

$$
G(K \sigma)=\left(\frac{K \kappa^{(\sigma)} \sigma g(\sigma)}{1+\sigma g(\sigma)}\right)\left\{1+O\left(\varepsilon_{2}(K, v)\right)\right\},
$$

provided that $v^{-1} K^{1 / 2} e^{-\alpha_{1} K} \leq K^{-1 / 2}$. Thus, if $G^{\prime}(0)>1$, the positive solution $s^{*}$ to $s=G(s)$ is such that $\sigma^{*}:=s^{*} / K$ satisfies

$$
\sigma^{*}=\left(\frac{\kappa^{\left(\sigma^{*}\right)} \sigma^{*} g\left(\sigma^{*}\right)}{1+\sigma^{*} g\left(\sigma^{*}\right)}\right)\left\{1+O\left(\varepsilon_{2}(K, v)\right)\right\} .
$$

The next lemma shows that omitting the order term when solving this equation makes little difference to the solution.

Lemma 5. Suppose, in addition to Conditions $1-5$, that $\kappa^{(0)} g(0)>1$. Then, whenever $v$ is small enough, the equation

$$
\frac{\kappa^{(\sigma)} g(\sigma)}{1+\sigma g(\sigma)}=1
$$

has a unique solution $\hat{\sigma}$ in $0<\kappa^{(0)}-1 / g(0)<\sigma<5 / 4$, and $\left|\sigma^{*}-\hat{\sigma}\right|=$ $O\left(\varepsilon_{2}(K, v)\right)$ if $G^{\prime}(0)>1$ and $K e^{-\alpha_{1} K} \leq v$. 
Proof. Write (3.17) as

$$
\sigma+\{1 / g(\sigma)\}-\kappa^{(\sigma)}=0
$$

noting that the left hand side is negative for $\sigma=0$ and exceeds $3 / 4$ for $\sigma=2$. From (3.3), $d \kappa^{(\sigma)} / d \sigma<1 / 2$; also, from (3.4) and (3.5),

$$
\left|\frac{d}{d \sigma}\left(\frac{1}{g(\sigma)}\right)\right| \leq\left(\frac{v}{\rho \gamma K}\right) \sum_{j \geq 1} c_{2}^{j}\left(\frac{\rho \gamma K}{b(0)}\right) \sum_{l=1}^{j} \frac{1}{l} \leq c_{3} v,
$$

for $c_{3}:=\{b(0)\}^{-1} \sum_{j \geq 1} c_{2}^{j}(1+\log j)<\infty$. Hence, for $c_{3} v<1 / 2$, the solution $\hat{\sigma}$ to $(3.17)$ in $(0,2)$ is unique. Similar considerations then show that any $\sigma^{*}$ satisfying

$$
\frac{\kappa^{(\sigma)} g(\sigma)}{1+\sigma g(\sigma)}=1+\varepsilon
$$

also satisfies $\left|\hat{\sigma}-\sigma^{*}\right| \leq c_{4} \varepsilon$ uniformly for all $|\varepsilon| \leq \varepsilon_{0}$ small enough; for instance, provided that $c_{3} v<1 / 8$, one can take $\varepsilon_{0}=\frac{1}{4} \wedge\left(\kappa^{(0)} g(0)-1\right)$ and

$$
1 / c_{4}=(1 / 10)\left(1-\varepsilon_{0}\right)\left\{7\left(1-\varepsilon_{0}\right)-4\right\},
$$

as follows by considering the $\sigma$-derivative of $\kappa^{(\sigma)} /\{\sigma+1 / g(\sigma)\}$.

Hence, if $\kappa^{(0)}$ and $g(0)=K \rho \gamma v^{-1}(1-\zeta)$ are such that $\kappa^{(0)} g(0)>1$, and if $G^{\prime}(0)>1$, approximating $\sigma^{*}$ by the solution $\hat{\sigma}$ of (3.17) usually entails little error. What is more, if $\kappa^{(0)} g(0)$ is big enough and $\varepsilon_{2}(K, v)$ small enough, then $G^{\prime}(0)>1$ is automatic. The following theorem collects these facts.

Theorem 3. Suppose that Conditions 1-5 hold, that $c_{3} v<1 / 8$, and that $\gamma, K$ and $v$ are such that $K e^{-\alpha_{1} K} \leq v \leq \eta_{1} / \log K$ and that $\kappa^{(0)} g(0)>1+\delta_{0}$ for some fixed $\delta_{0}>0$, where $\kappa^{(\sigma)}$ and $g$ are as in (3.1) and (3.14) respectively. Let $\hat{\sigma}$ be the solution to (3.17). Then the differential equations (1.2) have a non-trivial equilibrium solution $\pi$ provided that $\left|\varepsilon_{2}(K, v)\right| \leq \varepsilon_{0}$ for some $\varepsilon_{0}$ small enough. The solution $\pi$ satisfies

$$
\pi\{0\}=\pi^{\prime}\{0\}\left\{1+O\left(\varepsilon_{2}(K, v)\right)\right\},
$$

where $\pi^{\prime}\{0\}=\left(\frac{1}{1+\hat{\sigma} g(\hat{\sigma})}\right)$. Furthermore, defining $\pi^{\prime}:=\pi^{\prime}\{0\} \Delta_{\{0\}}+\left(1-\pi^{\prime}\{0\}\right) \pi^{0}$, where $\pi^{0}$ is as defined in (2.3) with $s=K \hat{\sigma}$, we haved $d_{T}\left(\pi, \pi^{\prime}\right)=O\left(\gamma \sqrt{K} \varepsilon_{2}(K, v)\right)$.

Proof. First, from (3.15), for $s=K \sigma$, we have

$$
\left|s^{-1} G(s)\left(\frac{\{1+\sigma g(\sigma)\}}{\kappa^{(\sigma)} g(\sigma)}\right)-1\right| \leq \phi \varepsilon_{2}(K, v),
$$

for some $\phi$ and for all $\gamma \leq \gamma_{0}, 0<\sigma \leq 2$ and $v \log K \leq \eta_{0}$. Now, since $\kappa^{(0)} g(0)>1+\delta_{0}$, it follows that

$$
\lim _{\sigma \downarrow 0}\left(\frac{\kappa^{(\sigma)} g(\sigma)}{\{1+\sigma g(\sigma)\}}\right)>1+\frac{1}{2} \delta_{0}>1,
$$


and hence that $s^{-1} G(s)>1$ for $s$ small enough, provided that $\varepsilon_{2}(K, v)<$ $\delta_{0} /\left\{\phi\left(4+2 \delta_{0}\right)\right\}$. Thus $G^{\prime}(0)>1$ so long as $\kappa^{(0)} g(0)>1+\delta_{0}$ and $K$ and $v$ satisfying the conditions of the theorem are such that $\varepsilon_{2}(K, v) \leq \varepsilon_{2}^{\prime}$, for some $\varepsilon_{2}^{\prime}=\varepsilon_{2}^{\prime}\left(\delta_{0}\right)>0$.

By Corollary 1, (3.6), (3.14) and (3.16), it follows that

$$
\pi\{0\}=\left(\frac{1}{1+\sigma^{*} g\left(\sigma^{*}\right)}\right)\left(1+O\left(\varepsilon_{2}(K, v)\right)\right) .
$$

Then

$$
\begin{aligned}
\left|\frac{d}{d s}\left\{\frac{1}{1+\sigma g(\sigma)}\right\}\right| & =\left|\frac{g(\sigma)+\sigma g^{\prime}(\sigma)}{(1+\sigma g(\sigma))^{2}}\right| \\
& \leq \frac{\{g(\sigma)\}^{-1}+\sigma\left|g^{\prime}(\sigma)\right|\{g(\sigma)\}^{-2}}{(\sigma+1 / g(\sigma))^{2}}
\end{aligned}
$$

The numerator is clearly bounded in $0 \leq \sigma \leq 2$; the denominator is uniformly bounded away from zero in any small neighbourhood of $\hat{\sigma}$, because $\sigma+1 / g(\sigma)$ has bounded derivative and $\hat{\sigma}+1 / g(\hat{\sigma})=\kappa^{(\hat{\sigma})}>3 / 4$. Hence the function $1 /(1+$ $\sigma g(\sigma))$ is uniformly Lipschitz near $\hat{\sigma}$, and the first part follows from Lemma 5 .

For the second, we just need the distance between the two distributions $\pi^{0\left(\sigma^{*}\right)}$ and $\pi^{0(\hat{\sigma})}$, which, to the given order, can be estimated using the approximations in Lemma 1 instead. Setting

$$
B:=B^{(\sigma)}=\frac{\kappa^{(\sigma)} b\left(\kappa^{(\sigma)}\right)+\rho \gamma \sigma}{\kappa^{(\sigma)}\left(d^{\prime}\left(\kappa^{(\sigma)}\right)-b^{\prime}\left(\kappa^{(\sigma)}\right)\right)+\rho \gamma \sigma / \kappa^{(\sigma)}},
$$

note that both $B^{(\sigma)}$ and $\kappa^{(\sigma)}$ have derivative uniformly of order $\gamma$ near $\hat{\sigma}$, in view of (3.3), and because both $b$ and $d$ are twice continuously differentiable. Now $d_{T V}(\widehat{\mathrm{Po}}(\lambda), \widehat{\mathrm{Po}}(\lambda+\delta)) \leq \delta\{\lambda+\delta\}^{-1}$, so that this element in the approximating distribution leads to a distance of order $O\left(\gamma \varepsilon_{2}(K, v)\right)$. However, the mean shift of $\left\lfloor K \kappa^{(\hat{\sigma})}\right\rfloor-\left\lfloor K \kappa^{\left(\sigma^{*}\right)}\right\rfloor$ is of order $O\left(K \gamma \varepsilon_{2}(K, v)\right)$, and $d_{T V}(\operatorname{Po}(\lambda)$, Po $(\lambda+$ $\delta)$ ) is genuinely of order $O\left(\delta\{\lambda+\delta\}^{-1 / 2}\right)$, so that $d_{T V}\left(\pi^{0(\hat{\sigma})}, \pi^{0\left(\sigma^{*}\right)}\right)$ is of order $O\left\{\gamma \sqrt{K} \varepsilon_{2}(K, v)\right\}$, as claimed.

Remark 4 . The proof of the theorem actually shows that $\pi$ and $\pi^{\prime}$ differ only by order $O\left(\gamma \varepsilon_{2}(K, v)\right)$, apart from a mean difference of $\left\lfloor K \kappa^{(\hat{\sigma})}\right\rfloor-\left\lfloor K \kappa^{\left(\sigma^{*}\right)}\right\rfloor$ between the positive parts of the distributions, this being of order $O\left(K \gamma \varepsilon_{2}(K, v)\right)$. However, since the means of the positive parts are of order $O(K)$, the relative error of this difference is also only of order $O\left(\gamma \varepsilon_{2}(K, v)\right)$.

\section{Numerical examples and discussion}

Equation (3.17) is still rather complicated, with $g(\sigma)$ defined in (3.14) and $\kappa^{(\sigma)}$ as in (2.1). However, if $\gamma$ is small, $\kappa^{(\sigma)}=1+O(\gamma)$ uniformly in $0 \leq \sigma \leq 2$. Then, from (3.19),

$$
1 / g(\sigma)=1 / g(0)+O(v)=v /\{K \rho \gamma(1-\zeta)\}+O(v) .
$$


Thus, instead of (3.17), we can replace (3.18) by the formula

$$
\hat{\sigma}=1-v /\{K \rho \gamma(1-\zeta)\}+O(v+\gamma),
$$

uniformly in $K \geq K_{0}, K e^{-\alpha_{1} K} \leq v \leq \eta_{1} / \log K$ and $K \rho \gamma v^{-1}(1-\zeta) \geq \delta_{0}$, for any $\delta_{0}>0$; here, $\zeta$ is as in (3.5). This approximation can readily be computed from the parameters of the process. Combining it with (3.13), one immediately obtains, in this approximation

$$
\pi^{(\hat{\sigma})}\{0\} \approx \frac{v b(0)}{K \rho \gamma(b(0)-d(0)-\gamma)} .
$$

For this quantity to be less than 1 , one needs

$$
\frac{K \rho \gamma}{\nu b(0)}(b(0)-d(0)-\gamma)>1 \text {. }
$$

This is the approximate threshold condition $\kappa^{(0)} g(0)>1$ as in Theorem 3, which reduces at our level of approximation to $g(0)>1$, because $\kappa^{(0)} \approx 1$ when $\gamma$ is small.

The approximation (4.2) also makes clear that, if this justification of Levins's model is to be used, and if $\pi_{e n}\{0\}$ is not to be close to 0 , then $K \rho \gamma / v$ cannot be too big. This in turn implies a rather small migration rate, especially in view of the fact that the approximations are proved with $v \log K$ small; the quantity $K \rho \gamma$ represents the overall rate of successful migrations from a typical occupied patch, and this cannot be of larger order than the (small) catastrophe rate $\nu$, without making $\pi_{e n}\{0\}$ very small, and thus unoccupied patches very rare.

We now present some numerical results for a simple case which has already been used in the literature: pure logistic growth, with density-dependence only in the death rate. Hence, we take $b(x) \equiv 1+r$ and $d(x)=1+r x$; setting $d(0)=1$ merely determines the time scale. For this example, the simplified threshold condition (4.3) becomes

$$
\frac{K \rho \gamma(r-\gamma)}{v(1+r)}>1
$$

which shows the typical feature that, for the metapopulation to persist, the dispersal rate $\gamma$ must lie in an intermediate region.

The first thing that we explored was how the exact threshold condition $G^{\prime}(0)>1$ depends on the parameters, and how well it can be approximated by simpler conditions such as (4.4). Now, from (1.7), the threshold condition can be written as $\gamma I_{1}>1 / \rho$, where

$$
I_{j}:=I_{j}(\gamma, r):=\int_{0}^{\infty} \mathbb{E}^{(j)} Z_{t}^{(0)} d t .
$$

The quantities $I_{j}$ satisfy the system of linear equations

$$
I_{j}=\left\{\lambda_{j} I_{j+1}+\mu_{j} I_{j-1}+j\right\} /\left\{\lambda_{j}+\mu_{j}+v\right\}, \quad j \geq 1,
$$



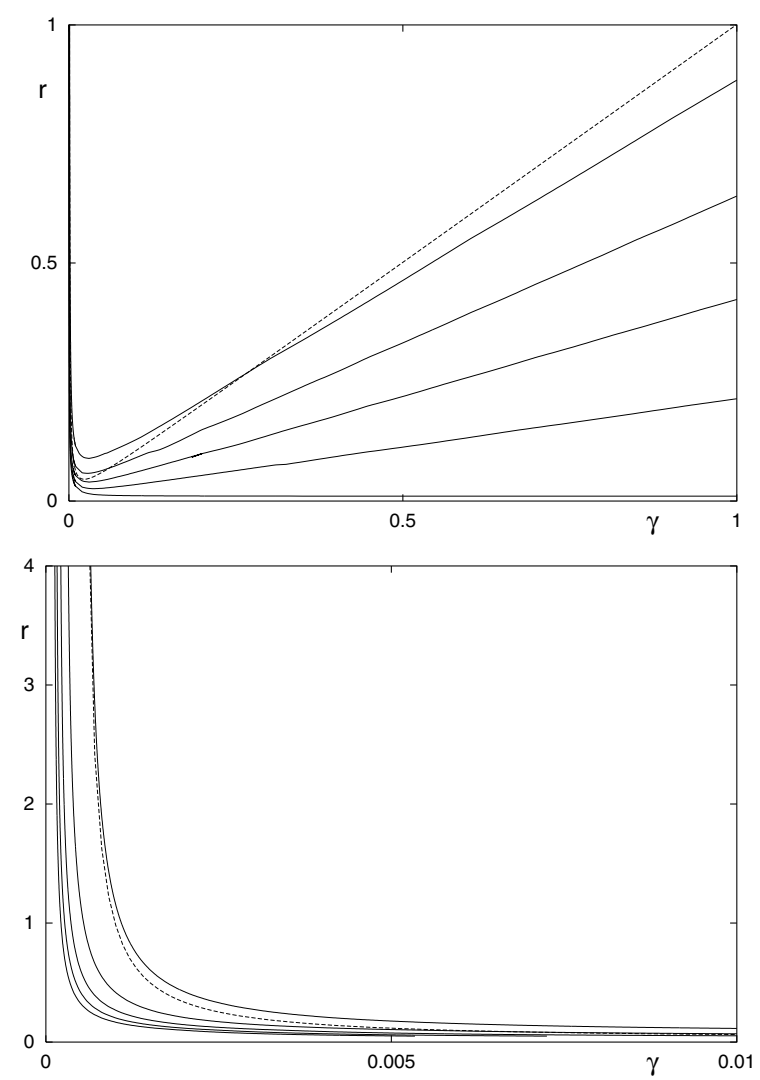

Fig. 1. Level curves $G^{\prime}(0)=1$ in the $(\gamma, r)$-plane. The different solid lines, from top to bottom, correspond to $\rho=0.2,0.4,0.6,0.8,1$; the dashed line is the curve obtained from (4.4) for $\rho=0.2$. Other parameter values are $K=100$ and $v=0.01$. The lower figure is a detail of the upper one.

with $I_{0}=0$, where, as in (2.2) with $s=0, \lambda_{j}=j(1+r)$ and $\mu_{j}=j(1+$ $r(j / K)+\gamma)$. Thus

$$
I_{j} \leq I_{j+1} \leq I_{j}+\left\{\left(q_{j}-p_{j}\right)(2+r(1+j / K)+\gamma)\right\}^{-1},
$$

where $p_{j}=\lambda_{j} /\left(\lambda_{j}+\mu_{j}\right), q_{j}=\mu_{j} /\left(\lambda_{j}+\mu_{j}\right)$ and the second inequality follows from a simple random walk comparison. This enables lower and upper estimates $I_{j}^{(M)}$ of $I_{j}$ to be determined, $1 \leq j \leq M$, by solving the finite set of linear equations (4.5) in which $1 \leq j \leq M-1$, together with an $M$-equation in which $I_{M+1}$ is replaced by either the lower or the upper bound from (4.6). For $M$ large enough, the lower and upper estimates are very close to each other, so that the computation of $\gamma I_{1}$ is reliable.

The curves in Fig. 1 show the persistence region in the $(\gamma, r)$-plane; they represent the $1 / \rho$ contours of the function $\gamma I(\gamma, r)$ for different values of $\rho \leq 1$. The 
overall shape of the level curves shows that persistence occurs only for intermediate values of $\gamma$ (unless $\rho=1$ ), as has already been found for this metapopulation model when the catastrophe rate $v=0$ [6].

It can also be seen that the approximation (only the one for $\rho=0.2$ is shown) $\frac{K \rho \gamma(r-\gamma)}{v(1+r)}=1$ works very well when $\gamma$ is very close to 0 , but quantitavely differs from (4.4) for larger $\gamma$. Indeed, it is clear that the approximation (4.4) requires $r>\gamma$, while, if $\rho$ is close to 1 , a metapopulation may also persist with $r<\gamma$. Note that Condition 5a is violated if $r \leq 4 \gamma$, so that in fact none of the approximations in this paper are justified unless $r>4 \gamma$; however, the conditions imposed in this paper are not necessary for the exact condition $G^{\prime}(0)>1$ for persistence to be true.

We now turn to the properties of the equilibrium distribution $\pi_{e n}$ in the persistence region. We begin by calculating $\pi^{(\sigma)}$ for any given $\sigma$. Once again, for any $M$, we can determine upper and lower bounds for the probabilities $\pi^{(\sigma)}\{j\}$, $0 \leq j \leq M$. First, we calculate bounds for the mean occupation times in states $1 \leq j \leq M$ between visits to the state 0 , by solving the equations

$$
\lambda_{j-1} x_{j-1}-\left(\lambda_{j}+\mu_{j}+v\right) x_{j}+\mu_{j+1} x_{j+1}=0, \quad 1 \leq j \leq M-1,
$$

with $x_{0}=(K \rho \gamma \sigma)^{-1}$, taking the upper boundary condition

$$
\lambda_{M-1} x_{M-1}=\left(\lambda_{M}+\mu_{M}+v\right) x_{M}
$$

for the lower bound $x^{(1)}$, and taking

$$
\lambda_{M-1} x_{M-1}=\left(\mu_{M}+v\right) x_{M}
$$

for the upper bound $x^{(2)}$; these equations correspond to processes which replace jumps from $M$ to $M+1$ by jumps to 0 and $M$, respectively. The quantities $\lambda_{j}$ and $\mu_{j}$ are as in (2.2). The sum $T^{(1)}:=\sum_{j=0}^{M} x_{j}^{(1)}$ is then a lower bound for the mean recurrence time for the state 0 , and

$$
T^{(2)}:=\sum_{j=0}^{M} x_{j}^{(2)}+x_{M}^{(2)} \lambda_{M} /\left(\mu_{M}-\lambda_{M}\right)
$$

is an upper bound, derived from a simple random walk comparison. Hence it follows that

$$
x_{j}^{(1)} / T^{(2)} \leq \pi^{(\sigma)}\{j\} \leq x_{j}^{(2)} / T^{(1)} \text { for } 0 \leq j \leq M .
$$

By choosing $M$ large enough, the upper and lower bounds can be brought as close as desired.

The value of the positive solution $s^{*}$ to the equation $s=G(s)$ is now found using the recursion

$$
s_{n+1}=G\left(s_{n}\right)=\sum_{j \geq 0} j \pi^{\left(s_{n} / K\right)}\{j\} .
$$


Table 1. Computation of some quantities of interest when the values of $K, \gamma$ and $v$ are those listed in the first three columns. In all cases, $r=1$ and $\rho=0.4$. The columns headed (3.17) and (4.1) refer to the corresponding approximations to $\sigma^{*}$; those headed (3.13) and (4.2) refer to approximations to $\pi_{e n}\{0\}$.

\begin{tabular}{rllllllll}
$K$ & \multicolumn{1}{c}{$\gamma$} & $\nu$ & $\sigma^{*}$ & $(3.17)$ & $(4.1)$ & $\pi_{e n}\{0\}$ & $(3.13)$ & $(4.2)$ \\
25 & 0.016 & 0.04 & 0.225 & 0.493 & 0.492 & 0.707 & 0.69 & 0.508 \\
50 & 0.004 & 0.02 & 0.361 & 0.502 & 0.498 & 0.586 & 0.579 & 0.502 \\
100 & 0.001 & 0.01 & 0.425 & 0.502 & 0.499 & 0.543 & 0.539 & 0.501 \\
200 & 0.00025 & 0.005 & 0.459 & 0.501 & 0.5 & 0.522 & 0.521 & 0.5 \\
400 & $6.25 \times 10^{-5}$ & 0.0025 & 0.478 & 0.501 & 0.5 & 0.512 & 0.511 & 0.5 \\
800 & $1.56 \times 10^{-5}$ & 0.00125 & 0.488 & 0.5 & 0.5 & 0.506 & 0.506 & 0.5
\end{tabular}

The inequality above shows that $G(K \sigma) \geq \sum_{j=0}^{M} j x_{j}^{(1)} / T^{(2)}$; a simple random walk comparison then shows that

$$
\begin{aligned}
& G(K \sigma) \leq \sum_{j=0}^{M} j x_{j}^{(2)} / T^{(1)} \\
& \quad+M x_{M}^{(2)}\left\{\lambda_{M} /\left(\mu_{M}-\lambda_{M}\right)\right\}\left\{1+\frac{K \rho \gamma \sigma}{M\{2+r(1+M / K)+\gamma\}}\right\} .
\end{aligned}
$$

The value of $M$ can also be chosen to ensure that the differences between these lower and upper bounds for $G(K \sigma)$ are small enough, for all $0 \leq \sigma \leq 2$. Using these numerical procedures, we were then able to compare $\sigma^{*}$ and some properties of $\pi_{e n}=\pi^{\left(\sigma^{*}\right)}$ with the corresponding approximations developed in this paper.

We started (see Table 1 ) by progressively increasing $K$, while decreasing $v$ and $\gamma$, so that $\pi_{e n}\{0\}$ would roughly remain constant. First of all (Figs. 2 and 3), we show the distribution $\pi_{e n}$ compared to the distributions $\pi^{\prime}$ and $\pi^{\prime \prime}$ presented in Corollary 1. It can be seen that, already for $K=100$ (Fig. 2), the Poisson approximation is remarkable: $\pi^{\prime \prime}$ is almost indistinguishable from $\pi^{\prime}$, and both closely resemble $\pi_{e n}$. For $K=800$ (Fig. 3), the three distributions are almost coincident.

We then considered in more detail how well $\pi_{e n}\{0\}$ and $\sigma^{*}$ are approximated. In addition to Table 1 , in which $K, v$ and $\gamma$ all vary in such a way that $v \log K \rightarrow 0$ and $K \rightarrow \infty$ (as required for our approximations to become exact), while keeping $\pi_{e n}\{0\}$ roughly constant, we give three other tables. In Table $4, K$ is kept constant at 100 , while $v$ decreases and $\gamma$ also, to keep $\pi_{e n}\{0\}$ roughly constant; here, comparing with Table 1, it can be seen that the value of $K$ has little effect on the quality of the approximations for $K \geq 100$. In Table 4, $v$ decreases but $\gamma$ is kept fixed, so that now $\pi_{e n}\{0\}$ decreases fast, but once again the quality of the approximations is unaffected; and in Table 4, $K$ is increased and $v$ decreased. In each table, we start from the values on the third line of Table 1 .

We can see that approximation (3.13) works remarkably well in all cases considered; indeed, we do not show computations of the approximation stated in Theorem 2, since it yields negligible improvements over (3.13) at a higher computational cost. Both of these approximations are computed with $\sigma=\sigma^{*}=K^{-1} s^{*}$, where $s^{*}$ is the exact solution to the fixed point equation $G(s)=s$. In contrast, 


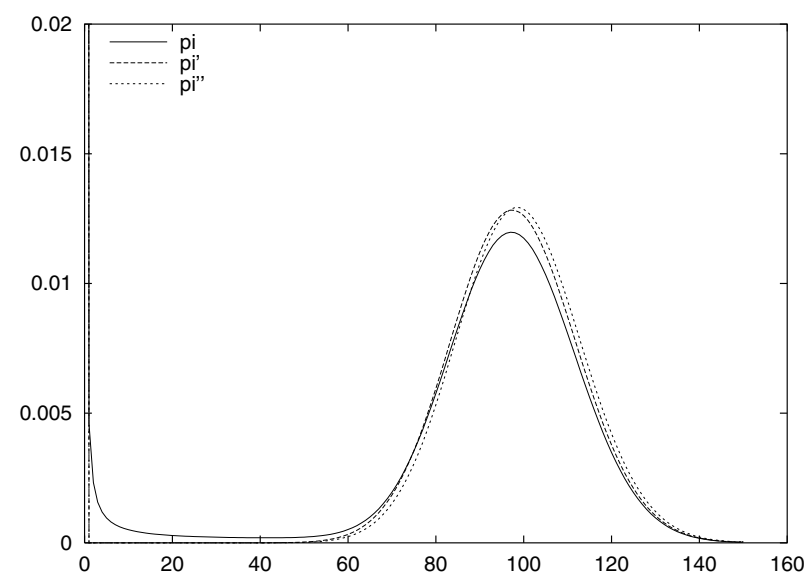

Fig. 2. Distributions $\pi_{e n}$ (labelled pi), $\pi^{\prime}$ (pi') and $\pi^{\prime \prime}$ (pi') for the parameter values in the third line of Table 1 . The value $\pi_{e n}\{0\} \approx 0.543$ is out of the scale.

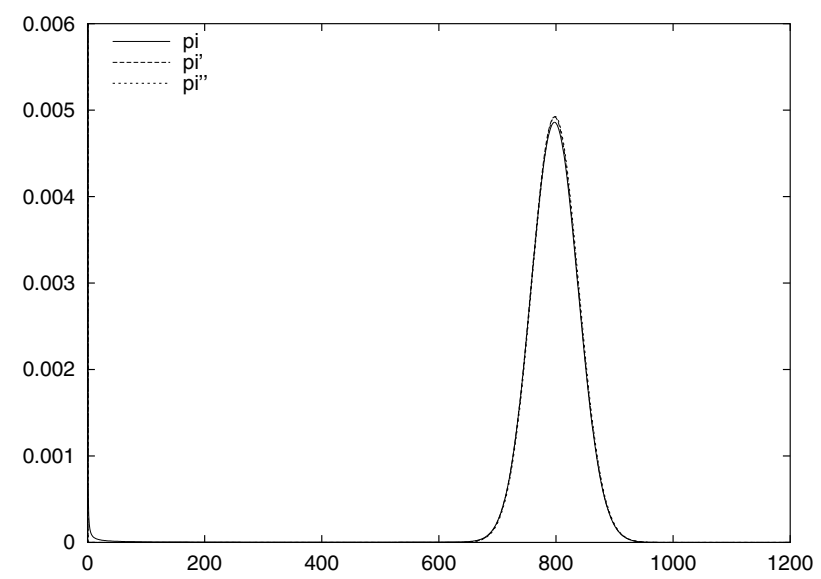

Fig. 3. Distributions $\pi_{e n}$ (labelled pi), $\pi^{\prime}$ (pi') and $\pi^{\prime \prime}$ (pi') for the parameter values in the last line of Table 1 . The value $\pi_{e n}\{0\} \approx 0.506$ is out of the scale.

formula (4.2), which uses the approximation $\hat{\sigma}$ from Lemma 5 and also (4.1) in its derivation, approaches the correct value $\pi_{e n}\{0\}$ rather fast in Table 1 , where the conditions $K \rightarrow \infty$ and $\gamma, v \rightarrow 0$ required for these approximations are satisfied, but only slowly in Table 4 , where $K$ is held fixed; in all cases, (3.13) performs much better.

That (4.2) performs less well is not surprising, inasmuch as the tables show that the approximation of the equilibrium value $\sigma^{*}$ obtained by solving (3.17) is not entirely satisfactory, and generally gives little improvement over simply computing (4.1). This can also be seen by looking at the approximation of the function $G$, which we plot for some of the combinations of parameter values given in Table 1. 
Table 2. Computation of some quantities of interest with decreasing $\gamma$ and $v$. In all cases, $r=1$ and $\rho=0.4$.

\begin{tabular}{lllllllll}
$K$ & \multicolumn{1}{c}{$\gamma$} & \multicolumn{1}{c}{$\nu$} & $\sigma^{*}$ & $(3.17)$ & $(4.1)$ & $\pi_{\text {en }}\{0\}$ & $(3.13)$ & $(4.2)$ \\
100 & 0.001 & 0.01 & 0.425 & 0.502 & 0.499 & 0.543 & 0.539 & 0.501 \\
100 & 0.0005 & 0.005 & 0.446 & 0.501 & 0.5 & 0.532 & 0.528 & 0.5 \\
100 & 0.00025 & 0.0025 & 0.457 & 0.501 & 0.5 & 0.527 & 0.522 & 0.5 \\
100 & 0.000125 & 0.00125 & 0.463 & 0.5 & 0.5 & 0.524 & 0.519 & 0.5 \\
100 & $6.25 \times 10^{-5}$ & 0.000625 & 0.466 & 0.5 & 0.5 & 0.523 & 0.518 & 0.5
\end{tabular}

Table 3. Computation of some quantities of interest with decreasing $v$. In all cases, $r=1$ and $\rho=0.4$.

\begin{tabular}{lllllllll}
$K$ & $\gamma$ & \multicolumn{1}{c}{$v$} & $\sigma^{*}$ & $(3.17)$ & $(4.1)$ & $\pi_{\text {en }}\{0\}$ & $(3.13)$ & $(4.2)$ \\
100 & 0.001 & 0.01 & 0.425 & 0.502 & 0.499 & 0.543 & 0.539 & 0.501 \\
100 & 0.001 & 0.005 & 0.701 & 0.752 & 0.75 & 0.264 & 0.261 & 0.25 \\
100 & 0.001 & 0.0025 & 0.84 & 0.876 & 0.875 & 0.13 & 0.128 & 0.125 \\
100 & 0.001 & 0.00125 & 0.909 & 0.938 & 0.937 & 0.0648 & 0.0636 & 0.0626 \\
100 & 0.001 & 0.000625 & 0.944 & 0.969 & 0.969 & 0.0323 & 0.0317 & 0.0313
\end{tabular}

Table 4. Computation of some quantities of interest with increasing $K$ and decreasing $\nu$. In all cases, $r=1$ and $\rho=0.4$.

$\begin{array}{lllllllll}K & \gamma & \nu & \sigma^{*} & (3.17) & (4.1) & \pi_{\text {en }}\{0\} & (3.13) & (4.2) \\ 100 & 0.001 & 0.01 & 0.425 & 0.502 & 0.499 & 0.543 & 0.539 & 0.501 \\ 200 & 0.001 & 0.005 & 0.838 & 0.877 & 0.875 & 0.128 & 0.127 & 0.125 \\ 400 & 0.001 & 0.0025 & 0.949 & 0.97 & 0.969 & 0.0305 & 0.0304 & 0.0313 \\ 800 & 0.001 & 0.00125 & 0.981 & 0.992 & 0.992 & 0.0072 & 0.00719 & 0.00782\end{array}$

The approximation of $G(s)$ given in Theorem 2 works reasonably well (left part of Fig. 4) with the parameter values used in Fig. 2 (moderately large $K$, moderately small $\gamma$ and $v$ ), very well (right part of Fig. 4) when they are all made more extreme as in Fig. 3, and also (left part of Fig. 5) when $\gamma$ is increased relative to the value for the lower part of Fig. 4; but rather poorly (right part of Fig. 5) when $K$ is large, but $\gamma$ and $v$ are only moderately small. The approximation (3.15) cannot do any better, since it is based on substituting the approximation (3.13) for $1-\pi^{(\sigma)}\{0\}$ in Theorem 2.

Expressed in terms of the result proved in Lemma 5, both $K^{-1 / 2}$ and $v \log K$ must become small to guarantee that $\hat{\sigma}$ approaches $\sigma^{*}$, and the difference between the curves in the two parts of Fig. 5 highlights the effect of increasing $v$. In terms of the underlying rationale of the approximations, the error arises from the fact that the distributions $\pi^{(\sigma)}$ not only have a point mass at 0 , in addition to the Poisson-like peak around $K \kappa^{(\sigma)}$, but also a decreasing part close to 0 , as can clearly be seen in Fig. 2 and also to some extent in Fig. 3. If one sums $\pi_{e n}\{i\}$ up to $J^{s}$, where $J^{s}$ is the minimal $j$ such that $\pi_{e n}\{j+1\}>\pi_{e n}\{j\}$, one obtains a value rather larger than $\pi^{(\hat{\sigma})}\{0\}$, even if the difference is still of order $O(v \log K)$. We found empirically 

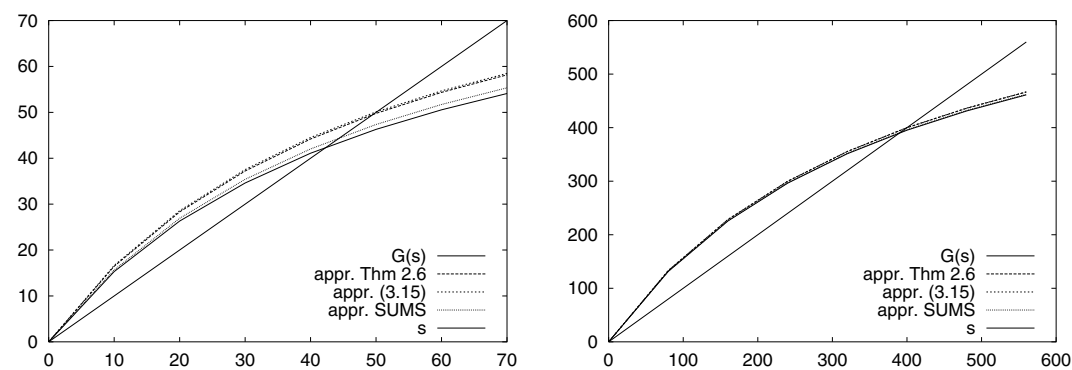

Fig. 4. The function $G(s)$, the approximation given in Theorem 2 (labelled appr. Thm. 2.6), the approximation (3.15) (appr. (3.15)) and the approximation (4.7) (appr. SUMS), together with the bisectrix. The points of crossing of the several curves with the bisectrix correspond to the values of $\sigma^{*}$ and its approximations, listed in Table 1. Left, the parameters are as in Fig. 2; right, as in Fig. 3.
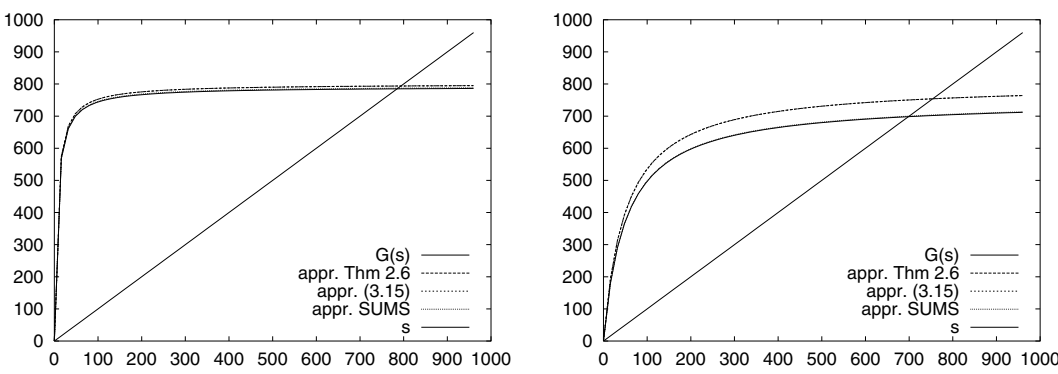

Fig. 5. The function $G(s)$ and its approximations, as in Fig. 4. Left, the parameter values are as in the last line of Table 4; right, they are the same, except that $v=0.01$.

that, by replacing the approximation $G(s) \approx K \kappa^{(\sigma)} \pi^{(\sigma)}\{0\}$ of Theorem 2 by

$$
G(s) \approx K \kappa^{(\sigma)} \sum_{j=0}^{J^{s}} \pi^{(\sigma)}\{j\}
$$

we consistently obtained a much better approximation to $G$.

Of course, computing the sum $\sum_{j=0}^{J^{s}} \pi^{(\sigma)}\{j\}$ involves first finding the stationary distribution $\pi^{(\sigma)}$, so that any computational saving in using approximations has been lost, whereas the qualitative advantages of the more accurate approximations over those given in this paper would seem to be slight. Thus it may be of interest to develop accurate approximations to $\sum_{i \leq J} \pi^{(\sigma)}\{i\}$ that do not require computation of $\pi^{(\sigma)}$. An approximation of this kind would be helpful when $K$ is large, but $v$ and $\gamma$ are only moderately small: for such parameters, the other approximations are very good, but that of $\sigma^{*}$ is not.

To conclude, we remark that Casagrandi and Gatto [6] present several approximations for a model very similar to the one considered here, based on the a priori 
assumption that the distribution of $p_{i}(t)$ is Poisson or negative binomial at each time $t$. Their main interest was in studying the parameter region in which the persistence condition $G^{\prime}(0)>1$ holds; it appears that, at least in this respect, their approximations perform rather well. It would be interesting to study the relations between their heuristically derived results and ours; and to see whether one can exploit the Poisson approximation shown in Theorem 1 to improve the approximation of the region $G^{\prime}(0)>1$.

\section{References}

1. Arrigoni, F.: Deterministic approximation of a stochastic metapopulation model. Adv. Appl. Probab. 35, 691-720 (2003)

2. Barbour, A.D.: Topics in Poisson approximation. In Stochastic Processes, Theory and Methods; Handbook of Statistics 19, Eds D. N. Shanbhag and C. R. Rao, Elsevier Science, (2001) pp. 79-115

3. Barbour, A.D., Pugliese, A.: Asymptotic behaviour of a metapopulation model. Università di Trento, Dip. Matematica, Preprint Series, Nr. 614 (2002)

4. Casagrandi, R., Gatto, M.: A mesoscale approach to extinction risk in fragmented habitats. Nature 400, 560-562 (1999)

5. Casagrandi, R., Gatto, M.: A persistence criterion for metapopulations. Theor. Pop. Biol. 61, 115-125 (2002)

6. Casagrandi, R., Gatto, M.: Habitat destruction, environmental catastrophes and metapopulation extinction. Theor. Pop. Biol. 61, 127-140 (2002)

7. Chesson, P.: Persistence of a Markovian population in a patchy environment. Z. Wahrscheinlich. 66, 97-107 (1984)

8. Diekmann, O., Heesterbeek, J.A.P., Metz, J.A.J.: On the definition and the computation of the basic reproduction ratio $R_{0}$ in models for infectious diseases in heterogeneous populations. J. Math. Biol. 28, 365-382 (1990)

9. Gyllenberg, M., Hanski, I.A.: Single-species metapopulation dynamics: a structured model. Theoret. Population Biol. 42, 35-61 (1992)

10. Gyllenberg, M., Metz, J.A.J.: On fitness in structured metapopulations. J. Math. Biol. 43, 545-560 (2001)

11. Hanski, I., Ovaskainen, O.: The metapopulation capacity of a fragmented landscape. Nature 404, 755-758 (2000)

12. Keeling, M.J.: Using individual-based simulations to test the Levins metapopulation paradigm. J. Anim. Ecology 71, 270-279 (2002)

13. Levins, R.: Some demographic and genetic consequences of environmental heterogeneity for biological control. Bull. Entomol. Soc. Am. 15, 237-240 (1969)

14. Metz, J.A.J., Gyllenberg, M.: How should we define fitness in structured metapopulation models? Including an application to the calculation of evolutionarily stable dispersal strategies. Proc. R. Soc. London B 268, 499-508 (2001)

15. Nee, S., May, R.M.: Dynamics of metapopulations: habitat destruction and competitive coexistence. J. Anim. Ecol. 61, 37-40 (1992) 